\title{
Developments in fractal geometry
}

\author{
Michael Barnsley • Andrew Vince
}

Received: 11 January 2013 / Revised: 10 April 2013 / Accepted: 27 May 2013 /

Published online: 6 June 2013

(C) The Author(s) 2013. This article is published with open access at SpringerLink.com

\begin{abstract}
Iterated function systems have been at the heart of fractal geometry almost from its origins. The purpose of this expository article is to discuss new research trends that are at the core of the theory of iterated function systems (IFSs). The focus is on geometrically simple systems with finitely many maps, such as affine, projective and Möbius IFSs. There is an emphasis on topological and dynamical systems aspects. Particular topics include the role of contractive functions on the existence of an attractor (of an IFS), chaos game orbits for approximating an attractor, a phase transition to an attractor depending on the joint spectral radius, the classification of attractors according to fibres and according to overlap, the kneading invariant of an attractor, the Mandelbrot set of a family of IFSs, fractal transformations between pairs of attractors, tilings by copies of an attractor, a generalization of analytic continuation to fractal functions, and attractor-repeller pairs and the Conley "landscape picture" for an IFS.
\end{abstract}

Keywords Fractal $\cdot$ Iterated function system

Mathematics Subject Classification (1991) 28A80

Communicated by Neil Trudinger.

\section{Barnsley ( $\varangle)$}

The Australian National University, Canberra, Australia e-mail: mbarnsley@aol.com; michael.barnsley@anu.edu.au

A. Vince

Department of Mathematics, University of Florida, Gainesville, FL, USA

e-mail: avince@ufl.edu 


\section{Introduction}

Metric spaces such as Euclidean space, the sphere, and projective space possess rich families of simple geometrical transformations $f: X \rightarrow X$. Examples are affine transformations of Euclidean space, Möbius and quadratic conformal mappings on the sphere, and projective transformations on projective space. The space and the mappings are simple to describe explicitly, and they are smooth.

In this review the basic object, denoted $\left\{X ; f_{1}, f_{2}, \ldots f_{N}\right\}$ and called an iterated function system (IFS), is a complete metric space $X$ together with a finite set of simple transformations on $X$. Let $H$ be the collection of nonempty compact subsets of $X$. Define $F: H \rightarrow H$ by $F(S)=\cup f_{n}(S)$ for all $S \in H$. Under fairly general conditions, the map $F$ has one or more attractors, an attractor being an attractive fixed-point of $F$. Although $X$ and the functions $f_{n}$ may be smooth, an attractor can be geometrically complicated and rough; that is, it may have features which are non-differentiable, or have a non-integer Hausdorff-Besicovitch dimension, or have a dense set of singularities. Attractors and transformations between them comprise the principal objects of study in deterministic fractal geometry. They can be arcs of graphs of wavelets, Julia sets, Sierpinski triangles, or geometrical models for intricate biological structures such as leaf veins. Many textbooks use pictures of such objects to illustrate the idea of a fractal; Fig. 1 illustrates a few familiar IFS fractals, and also some newer fractal objects associated with simple geometrical IFSs.

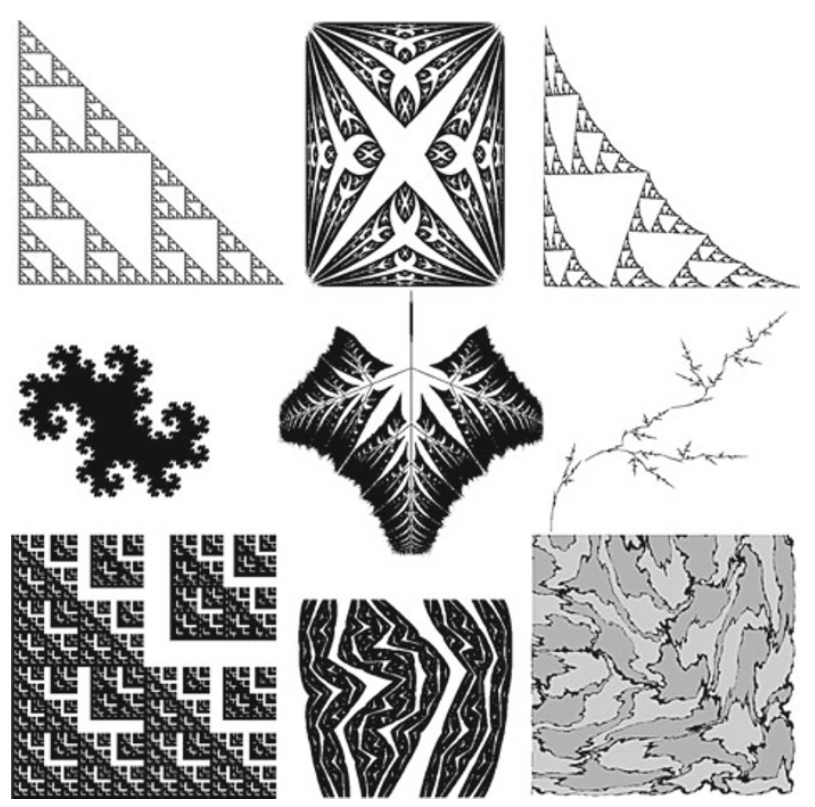

Fig. 1 Fractal objects associated with IFSs on $\mathbb{R}^{2}$. The first column illustrates some familiar attractors of affine IFSs; the second column illustrates attractors of bi-affine IFSs; the top two members of the third column are attractors of projective IFSs; the bottom right picture illustrates the result of applying a fractal homeomorphism, constructed using two affine IFSs, to a regular tiling by equilateral triangles 
Following the publication in 1983 of Benoit Mandelbrot's book The Fractal Geometry of Nature [88], there has been a steadily increasing interest in the use of nondifferentiable structures to model diverse natural objects and processes. In [20] $\mathrm{H}$. Furstenberg observes that fractals have fundamentally changed the way that geometers look at space. For some, there has been a shift in viewpoint, away from the study of smooth structures such as differentiable manifolds to the study of rough non-differentiable objects such as fractal attractors of smooth dynamical systems. The rough objects are described in terms of the smooth systems that generate them. A key example is an attractor described in terms of the IFS that generates it. Questions regarding the topology (connectedness for example), geometry (Hausdorff dimension for example), invariant measures and other properties of the fractal objects are investigated, to reveal their relationships to the smooth objects that generate them.

Using these relationships, fractal objects can be used to model or approximate rough real-world data, from stock-market traces to turbulent wakes and cloud boundaries. In physics, for example, fractal geometry has played a role in mapping the seabed and in modelling the effect of the rough surfaces of small particles on heat absorption by the atmosphere [42]. In engineering, fractal modelling has been successfully applied to image compression [14]. In multimedia, fractal modelling can be used for real-time image synthesis [97]. There are many more such examples, see [20].

Some fractal objects are self-referential. A self-referential object $A$ is a set or measure that can be defined in terms of a finite collection of geometrical transformations applied to $A$. For example, an attractor $A$ of $F: H \rightarrow H$ is self-referential since $A=\cup f_{n}(A)$. According to a definition by Mandelbrot [87, p. 15], a fractal is a set for which the Hausdorff-Besicovitch dimension strictly exceeds the topological dimension. Note, however, that the attractor of an IFS may not have this property and may, in fact, be a classical geometric object such as a line segment or polygon.

Although fractals receive considerable current attention, they are a newcomer to the history of geometry and to the task of describing physical objects. Fractal geometry is an area of mathematics that is under construction. In his much cited 1981 paper [65], John Hutchinson formulated the concept of a contractive iterated function system, although not with that name, to unify and make rigorous, using geometric measure theory, some of the key ideas in Mandelbrot's book [87]. Related precursors to the concept of an attractor of a contractive system can be found, for example, in the work of Nadler [94] and Williams [125], and ideas concerning associated invariant measures can be found in $[52,99]$.

This paper is a survey of some recent trends in the subject of iterated function systems. The focus is two-fold. First, to emphasize new structural properties of affine, projective, analytic, and even more general non-conformal IFSs, adding to older publications concentrating on similitude and conformal IFSs. Second, to concentrate on the core of the subject- the role of contractivity on the existence of an attractor, addressing the points of an attractor, transformations between attractors, dynamical properties of an IFS, and tilings by copies of an attractor. Although these notions are at the historical foundation of the subject, new and exciting concepts, such as phase transition and fractal continuation, have recently come to light.

Section 2 provides definitions and notation associated with an IFS and its attractors. Examples of similitude, affine, projective and Möbius IFSs are given. Some concepts, 
such as an attractor-repeller pair, are introduced informally in this section, but are defined formally in later sections. The definition of an attractor of an IFS that is used in this review is in keeping with recent work in the area, and is a generalization of the concept for contractive systems.

In Sect. 3 the notion of a contractive IFS $\mathcal{F}=\left\{X ; f_{1}, f_{2}, f_{N}\right\}$ is defined, followed by Hutchinson's theorem stating that a contractive IFS has a unique attractor. This theorem can be viewed as a generalization of Banach's contraction mapping theorem, generalizing from the existence of an attractive fixed point of a single function to the existence of an attractor of a finite set of functions. It is known, under quite general conditions, that if a transformation on a metrizable space possesses a unique fixed point, then there exists a metric with respect to which the transformation is contractive. When does an analogous situation hold for an IFS with a unique attractor? Recent work has shown that contractivity is a necessary condition for the existence of an attractor for some basic types of IFSs, such as affine, projective and Möbius IFSs.

Section 4 contains recent advances concerning the applicability of the chaos game, one of two main algorithms for drawing an accurate approximation to an attractor of an IFS. The two approaches are the deterministic algorithm (illustrated in Fig. 2) and the more efficient chaos game algorithm [19]. It has recently been proved that the chaos game algorithm converges to the attractor under general conditions.

The joint spectral radius $\rho(\mathcal{F})$ of the linear parts of an affine IFS $\mathcal{F}$ plays a crucial role in determining whether or not $\mathcal{F}$ possesses an attractor. This role is explained in Sect. 5. Considering $\rho(\mathcal{F})$ as a parameter, for linear IFSs there is a dramatic phase transition at the precise value of $\rho(\mathcal{F})$ at which the IFS changes from not having an attractor to having a trivial attractor. The fractal figure that emerges at this phase transition is the main subject of Sect. 5.

For an IFS consisting of $N$ functions, strings in the alphabet $\{1,2, \ldots, N\}$ can be associated with points in an attractor of the IFS. This leads to the topic of addresses and coordinate and coding maps, which are the subject of Sect. 6. Mentioned in Sect. 6.1 is Kieninger's classification of attractors according to their fibres and Kameyama's fundamental question: When does the existence of a coding map ensure the existence of a contractive metric? In Sect. 6.2 we define the recently introduced notion of a section of a coordinate map, whereby each point on an attractor is assigned a unique address. Sections of coordinate maps play a key role in the construction of transformations between attractors, which is the subject of Sect. 9 .

In Sect. 7, attractors of IFSs are classified according to how the images of the attractor, under the functions of the IFS, overlap. This leads to the notion of the kneading invariant and what it implies regarding the topology of an attractor.

The subject of Sect. 8 is the Mandelbrot set of a certain affine family $\mathcal{F}_{\lambda}$ of IFSs, an analog to the classical Mandelbrot set for $z \mapsto z^{2}+\lambda$. The Mandelbrot set is the set of those $\lambda \in \mathbb{C}$ for which $\mathcal{F}_{\lambda}$ possesses a connected attractor.

While properties of classical geometrical transformations (for example affine, conformal, projective) are well known, much less is known about their rough counterparts (for example affine, conformal, and projective fractal transformations). Transformation between fractals is discussed in Sect. 9.

Tilings of the plane, and more generally Euclidean space, have been constructed from attractors of an IFS since the 1980s. Section 10 provides a new and unified 

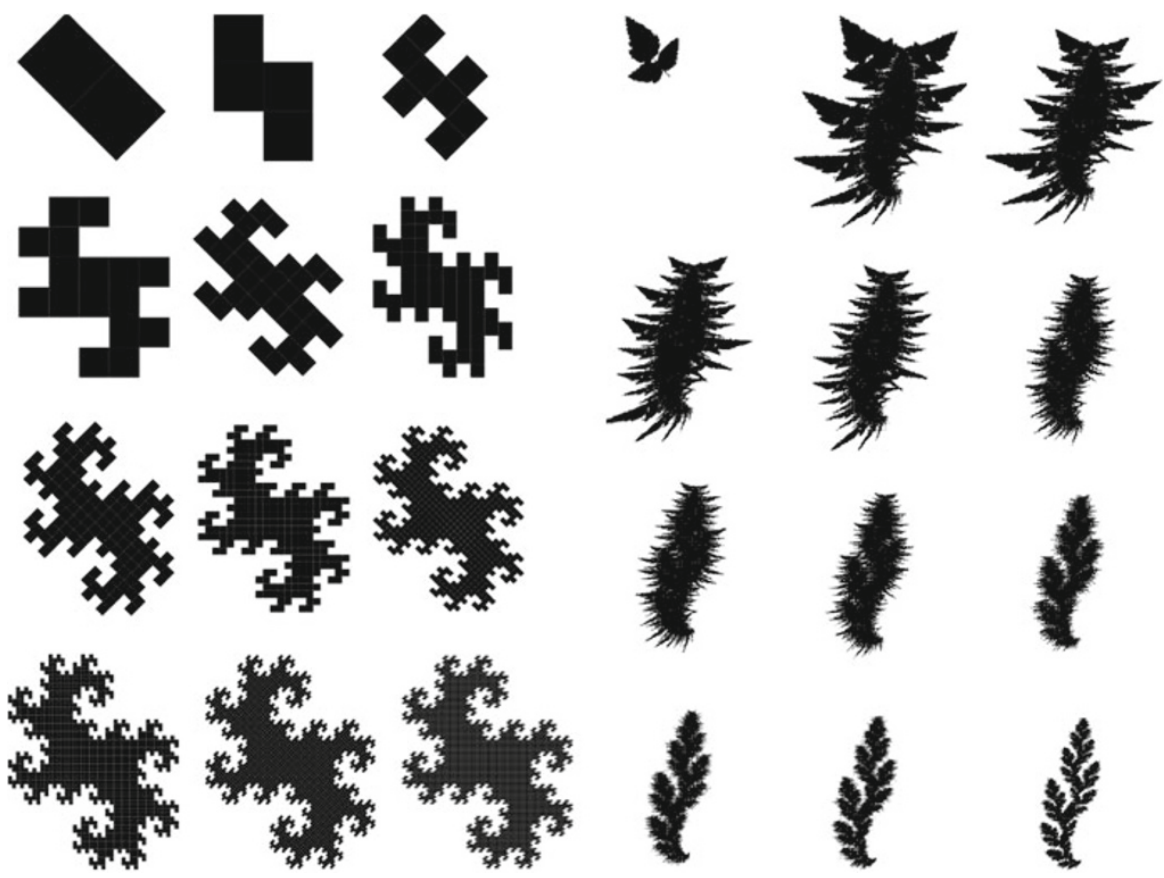

Fig. 2 This figure illustrates iterates of a Hutchinson operator, in two cases. In the first case the IFS comprises two similitudes, as in Eq. (2.1); the first three columns show successive iterates, applied to the rectangular set at top left, converging towards a discretized version of the twindragon fractal, at the bottom of the third column. In the second case the IFS comprises two affine maps that are not similitudes. Iterates of the associated Hutchinson operator, applied to a leaf-like set, are illustrated in the fourth, fifth and sixth columns

approach to such tiling_-generating both the classical self-similar IFS tilings and new tilings.

Fractal functions, introduced in the form considered here in 1986, have applications ranging from image compression to modeling brain waves. Fractal continuation of such functions, an analog to classical analytic continuation, is the subject of Sect. 11.

Based on the work of Conley on the dynamics of a single function and on the work of McGehee and Wiandt on iterated closed relations, a generalization of the notion of an attractor of an IFS is given in Sect. 12. This leads to the existence of attractorrepeller pairs, a characterization of the set of chain recurrent points of an IFS in terms of such pairs, and a "landscape picture" for an IFS.

This paper is not intended to be exhaustive. Many fascinating topics are omitted, and there is likely a bias towards the interests of the authors. In particular, "random fractals", "statistically self-similar measures", and related probabilistic entities are not discussed; our interest is in deterministic fractal geometry, where the information needed to describe the fractal object is low, and the geometrical complexity may appear to be high. Another topic not discussed is $V$-variable fractals and superfractals [16,2628]. These entities serve several purposes; they provide a bridge between deterministic fractal geometry and random fractal geometry, and they enable the construction of rich 
simple geometrical families of fractal objects. Fractal dimensions are only touched on in this survey because there is already an ample literature of the subject.

\section{IFSs and their attractors, basins, and dual repellers}

In this section we define and provide examples of some fundamental notions. In so doing we introduce the concept of an attractor-repeller pair that is explained in more detail in Sect. 12, which deals with Conley's "landscape picture" for an IFS.

Definition 2.1 An iterated function system (IFS) is a topological space $X$ together with a finite set of continuous functions $f_{n}: X \rightarrow X, n=1,2, \ldots, N$.

We use the notation

$$
\mathcal{F}=\left\{X ; f_{1}, f_{2}, f_{N}\right\}
$$

to denote an IFS. Other more general definitions of an IFS are used in the literature; for example, the collection of functions in the IFS may be infinite, see for example [71], or the functions may themselves be set-valued [76,79]. However, throughout this review, except where otherwise stated, $N$ is a finite positive integer and $X$ is a complete metric space. This fits with our philosophy that the system is geometrically simple. By slight abuse of terminology, we use the same symbol $\mathcal{F}$ for the IFS and for the set of functions in the IFS.

Let $H=H(X)$ denote the collection of nonempty compact subsets of $X$ and define the Hutchinson operator $F: H \rightarrow H$ by

$$
F(S)=\bigcup_{f \in \mathcal{F}} f(S)
$$

for all $S \in H$. By further slight abuse of notation we also treat $F$ as a map $F: 2^{X} \rightarrow$ $2^{X}$, where $2^{X}$ denotes the collection of all subsets of $X$. For $S \subset X$, define $F^{0}(S)=S$ and let $F^{k}(S)$ denote the $k$-fold composition of $F$ applied to $S$, namely, the union of $f_{i_{1}} \circ f_{i_{2}} \circ \cdots \circ f_{i_{k}}(S)$ over all finite words $i_{1} i_{2} \cdots i_{k}$ of length $k$.

Let $d:=d_{X}$ be the metric on $X$, and let $d_{H}:=d_{H(X)}$ be the corresponding Hausdorff metric. The Hausdorff metric is defined on the space $H(X)$ by

$$
d_{H}(S, T)=\min \{\delta \geq 0: S \subset B(T, \delta), T \subset B(S, \delta)\}
$$

for all $S, T \in H$, where

$$
B(S, \delta)=\left\{x \in X: d_{X}(x, s) \leq \delta \quad \text { for some } s \in S\right\}
$$

Throughout we assume that the topology on $H$ is the one induced by $d_{H}$. Key facts, discussed in [63], are that $\left(H, d_{H}\right)$ is a complete metric space because $(X, d)$ is complete, and that if $(X, d)$ is compact then $\left(H, d_{H}\right)$ is compact. 
Definition 2.2 An attractor of the IFS $\mathcal{F}$ is a set $A \in H(X)$ such that

(1) $F(A)=A$ and

(2) there is an open set $U \subset X$ such that $A \subset U$ and $\lim _{k \rightarrow \infty} F^{k}(S)=A$, for all $S \in H$ with $S \subset U$, where the limit is with respect to the Hausdorff metric on $H$.

We remark that, since $X$ is a complete metric space, we have $F: H(A) \rightarrow H(A)$ is continuous (w.r.t. the Hausdorff metric) see [30]. So (1) in Definition 2.2 is superfluous. But we include (1) because it is key in more general settings, such as the one considered in Sect. 12.

Definition 2.3 The basin $B$ of an attractor $A$ of the IFS $\mathcal{F}$ is the largest open set $U$ such that (2) in Definition 2.2 is true.

Definition 2.4 The dual repeller of an attractor $A$ of the IFS $\mathcal{F}$ is the set $A^{*}:=X \backslash B$ where $B$ is the basin of $A$.

Examples of attractors and their dual repellers appear below. The notion of a dual repeller arises as follows.

Definition 2.5 The IFS $\mathcal{F}=\left\{X ; f_{1}, f_{2}, \ldots, f_{N}\right\}$ is said to be invertible when $f_{n}$ : $X \rightarrow X$ is a homeomorphism for all $n$. If $\mathcal{F}$ is invertible, then the IFS

$$
\mathcal{F}^{-1}:=\left\{X ; f_{1}^{-1}, f_{2}^{-1}, \ldots, f_{N}^{-1}\right\}
$$

is called the inverse IFS of $\mathcal{F}$.

Under reasonable conditions described in Sect. 12, an attractor of $\mathcal{F}^{-1}$ is a dual repeller of an attractor of $\mathcal{F}$.

If $X=\mathbb{R}^{M}$ and the functions in $\mathcal{F}$ are affine functions of the form $f(x)=L x+a$, where $L$ is an $M \times M$ matrix and $a \in \mathbb{R}^{M}$, then $\mathcal{F}$ is called an affine IFS. If the functions of $\mathcal{F}$ are similitudes (i.e. affine with $L=s Q$ where $Q$ is orthogonal and $s \in(0,1)$,$) then \mathcal{F}$ is called a similitude IFS.

An example of a similitude IFS is

$$
\mathcal{F}=\left\{\mathbb{R}^{2} ; f_{1}(x, y)=\left(\frac{x-y}{2}, \frac{x+y}{2}\right), f_{2}(x, y)=\left(\frac{x-y}{2}+1, \frac{x+y}{2}\right)\right\} \text {. }
$$

The IFS (2.1) has a unique attractor because it is contractive; see Theorem 3.2. This attractor $A$, approximated by the bottom picture of the third column of Fig. 2, is called the twindragon. The basin of $A$ is $B=\mathbb{R}^{2}$, and the dual repeller is the empty set, $A^{*}=\emptyset$. The first three columns of Fig. 2 also demonstrates condition (2) in Definition 2.2 because they show the successive iterates $F^{k}(S)$ for $k=1,2, \ldots, 12$, where $S$ is the rectangle at the upper left. If, in this example, we change the space $X$ to be $\mathbb{R}^{2} \cup\{\infty\}$, the one point compactification of $\mathbb{R}^{2}$, where $\infty$ is "the point at infinity", and we define $f_{n}(\infty)=\infty$, then the basin is no longer the whole space and the dual repeller is $A^{*}=\{\infty\}$. Remarks analogous to those mentioned in this paragraph hold, 
in fact, for all similitude IFSs. Note that $S:=\mathbb{R}^{2} \cup\{\infty\}$ can be represented as a sphere (with infinity at say the north pole) and $\mathbb{R}^{2}$ is embedded in $S$ by stereographic projection. Then $\mathbb{R}^{2}$ and $S$ are both metric spaces, $\mathbb{R}^{2}$ with the Euclidean metric and $S$ with the spherical metric, and the two metrics give the same topology when restricted to $\mathbb{R}^{2}$. Both the $\mathbb{R}^{2}$ case and $\mathbb{R}^{2} \cup\{\infty\}$ case, of the IFS (2.1), are invertible. In the $\mathbb{R}^{2}$ case the affine IFS $\mathcal{F}^{-1}$ does not possess an attractor. In the $\mathbb{R}^{2} \cup\{\infty\}$ case the IFS $\mathcal{F}^{-1}$ has a unique attractor $A^{*}=\{\infty\}$, the dual repeller of the attractor $A$ of $\mathcal{F}$.

If $X=\mathbb{R} \mathbb{P}^{n}$, real $n$-dimensional projective space, and the functions in the IFS are projective functions of the form $f(x)=L x$, where $L$ is an $(n+1) \times(n+1)$ matrix and $x$ is given by homogeneous coordinates, then $\mathcal{F}$ is called a projective IFS. An example of a projective IFS is

$$
\mathcal{F}=\left\{\mathbb{R} \mathbb{P}^{2} ; f_{1}(x)=\left(\begin{array}{rrr}
41 & -19 & 19 \\
-19 & 41 & 19 \\
19 & 19 & 41
\end{array}\right) x, \quad f_{2}(x)=\left(\begin{array}{rcc}
-10 & -1 & 19 \\
-10 & 21 & 1 \\
10 & 10 & 10
\end{array}\right) x\right\}
$$

This IFS (2.2) possesses a unique attractor $A$, illustrated in Fig. 3. This attractor, which is the union of a non-denumerable set of lines, is illustrated using a disk, with antipodal points identified, to represent $\mathbb{R P}^{2}$. This image was computed using the chaos game algorithm, discussed in Sect. 4. The corresponding dual repeller $A^{*}$ is also shown: it is a spiral Cantor set in green, disjoint from the attractor. The basin of $A$ is $\mathbb{R} \mathbb{P}^{2} \backslash A^{*}$. The IFS (2.2) is invertible and its inverse $\mathcal{F}^{-1}$ possesses a unique attractor, equal to the dual repeller of $\mathcal{F}$, the spiral Cantor set to which we have just referred. The dual repeller of $\mathcal{F}^{-1}$ is the attractor $A$ of $\mathcal{F}$; and the basin of the attractor $A^{*}$ of $\mathcal{F}^{-1}$ is $\mathbb{R P}^{2} \backslash A$.
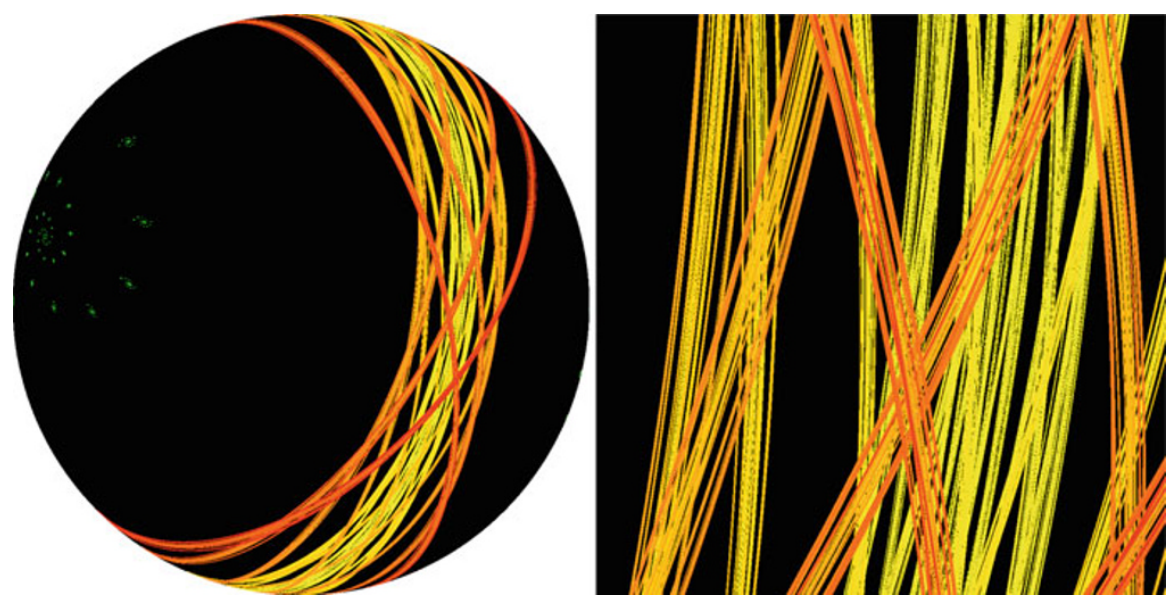

Fig. 3 The image on the left illustrates the attractor, in red, orange and yellow, and the dual repeller, in green, of the projective IFS (2.2), using a disk, with antipodal points identified, to represent the projective plane (black). The image on the right illustrates a zoom on the attractor. The colours help to distinguish lines in the attractor (color figure online) 
Fig. 4 The unique attractor (red) and dual repeller (black) of a certain Möbius IFS consisting of two transformations acting on the Riemann sphere (color figure online)

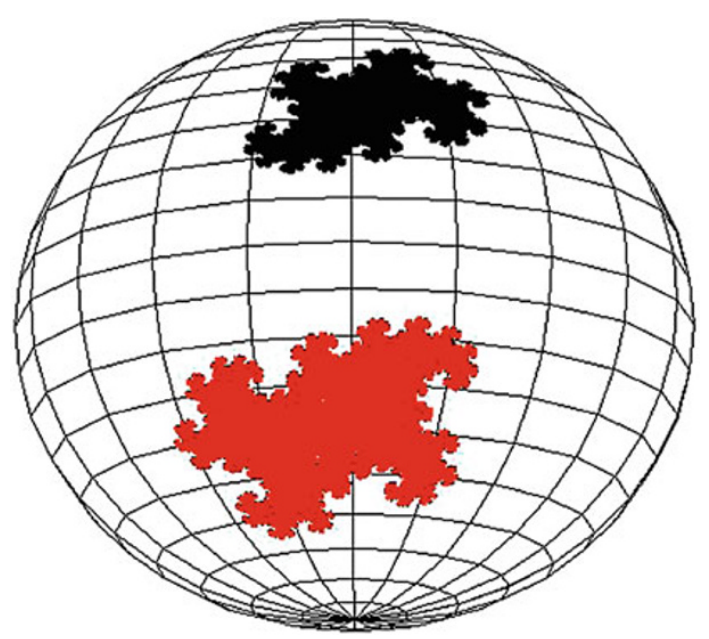

If $X=\widehat{\mathbb{C}}=\mathbb{C} \cup\{\infty\}$, the extended complex plane, and the functions are Möbius functions of the form $f(z)=\frac{a z+b}{c d+d}$, where $a d-b c \neq 0$ and $a, b, c, d$ are complex numbers, then $\mathcal{F}$ is called a Möbius IFS. Möbius functions may equivalently be considered to act on the Riemann sphere or the complex projective line. The unique attractor $A$ (red) and the dual repeller $A^{*}$ (black) for a certain Möbius IFS $(N=2)$ on the Riemann sphere are illustrated in Fig. 4 . The basin of the attractor is the complement of the dual repeller. In this case the dual repeller is the attractor of the inverse IFS, and the attractor is the dual repeller of the inverse IFS. For further details on such Möbius examples see [124].

\section{Contractive IFSs}

When does an IFS $\left\{X ; f_{1}, f_{2}, \ldots, f_{N}\right\}$ possess an attractor? We begin with the observation that if an IFS is contractive (defined below), then it possesses a unique attractor $A$, with the basin $B=X$, and dual repeller $A^{*}=\emptyset$. Then we make the following points:

(1) Contractivity is not integral to the existence of an attractor.

(2) Contractivity is integral to the existence of an attractor for affine, Möbius, and many projective IFSs.

Definition 3.1 A function $f:\{X\} \rightarrow\{X\}$ is a contraction with respect to a metric $d$ if there exists $\lambda \in[0,1)$ such that

$$
d(f(x), f(y)) \leq \lambda d(x, y)
$$

for all $x, y \in X$. An IFS $\mathcal{F}$ on a metric space $(X, d)$ is contractive if there is a metric $\widehat{d}$, inducing the same topology on $X$ as the metric $d$, with respect to which the functions in $\mathcal{F}$ are contractions. 
The following theorem is a generalization of the Banach contraction mapping theorem, from one function to finitely many functions.

Theorem 3.2 (Hutchinson) If $\mathcal{F}$ is a contractive IFS on a nonempty complete metric space $(X, d)$, then $\mathcal{F}$ has a unique attractor $A$ and the basin of $A$ is $X$.

Remark 1 There are various versions of a converse to Banach's fixed point theorem in the literature, for example $[44,55,67,80]$. However, the converse to Hutchinson's theorem is false. There exist non-contractive IFSs that have a unique attractor; for example, the IFS $\mathcal{F}$ in (2.2) has a unique attractor $A$, but there is no metric on any complete neighborhood $Y$ of $A$, generating the same topology as that of $\mathbb{R P}^{2}$ restricted to $Y$, such that $\mathcal{F}$ restricted to $Y$ is contractive.

Theorem 3.4 below addresses the role of contractivity, not only in the affine case, but also in the projective and Möbius cases. For a proof of the theorem, see [4] for the affine case, [34] for the projective case, and [124] for the Möbius case. The proof of the uniqueness result, Theorem 3.3, can also be found there. In the projective case, recall that a hyperplane in $n$-dimensional real projective space is an $(n-1)$-dimensional subspace. A set $S \subset \mathbb{R P}^{n}$ is said to avoid a hyperplane $\mathcal{H}$ if $S \cap \mathcal{H}=\emptyset$. In the Möbius case, recall that the extended complex plane $\widehat{\mathbb{C}}$ is essentially equivalent to the Riemann sphere via stereographic projection. For the Möbius case, the metric is the round metric on the sphere. Throughout, if $S \subset X$ then $\bar{S}$ and $S^{\circ}$ denote, respectively, the closure and the interior of $S$.

Theorem 3.3 An affine, Möbius, or projective IFS can have at most one attractor.

Theorem 3.4 (1) An affine IFS $\mathcal{F}=\left\{\mathbb{R}^{n} ; f_{1}, f_{2}, \ldots, f_{N}\right\}$ has an attractor if and only $\mathcal{F}$ is contractive.

(2) A Möbius IFS $\mathcal{F}=\left\{\widehat{\mathbb{C}} ; f_{1}, f_{2}, \ldots, f_{N}\right\}$ has an attractor $A \neq \widehat{\mathbb{C}}$ if and only there is a nonempty open set $U$ such that $\bar{U} \neq \widehat{\mathbb{C}}$ and $\mathcal{F}$ restricted to $U$ is contractive.

(3) A projective IFS $\mathcal{F}=\left\{\mathbb{R P P}^{n} ; f_{1}, f_{2}, \ldots, f_{N}\right\}$ has an attractor that avoids some hyperplane if and only if there is a nonempty open set $U$ such that $\mathcal{F}$ is contractive on $\bar{U}$.

The attractor of the IFS (2.2) is a union of hyperplanes (lines). Since the intersection of two lines in the projective plane is nonempty, the attractor avoids no hyperplane, and, by Remark 1, this shows that the condition in part (3) of Theorem 3.4 cannot be removed.

An interesting aspect of Theorem 3.4, that also makes it somewhat difficult to prove, is that, in general, the IFS is not necessarily contractive with respect to the standard metric, even locally. In the affine case on $\mathbb{R}^{n}$, an IFS with an attractor is always contractive with respect to a Minkowski metric, i.e. a metric of the form

$$
d_{C}(x, y)=\|x-y\|_{C}
$$

where

$$
\|x\|_{C}=\inf \{\lambda \geq 0: x \in \lambda C\}
$$


and where $C$ is a centrally symmetric convex body. In the projective case, an IFS with an attractor is always contractive with respect to a (modified) Hilbert metric. For a convex body $C \subset \mathbb{R} P^{n}$, the Hilbert metric $d_{C}$ on the interior $C^{o}$ is defined in terms of the cross ratio as

$$
d_{C}(x, y):=\log R(a, b, x, y)=\log \left(\frac{|a y||b x|}{|a x||b y|}\right)
$$

where $a$ and $b$ are the points where the line joining $x$ and $y$ intersects the boundary of $C$. In the Möbius case, an IFS with an attractor is always contractive on some open set $U$ with respect to the newly discovered metric on $\widehat{\mathbb{C}}$ :

$$
d_{U}(x, y)=\max _{z \notin U} \log \frac{|z-x|}{|z-y|}+\max _{z \notin U} \log \frac{|z-y|}{|z-x|} .
$$

In each case, affine, projective, and Möbius, an IFS with an attractor is also contractive in a topological sense. This give rise to Theorem 3.6 below, a companion to Theorem 3.4. The proof appears in the same references cited for Theorem 3.4.

Definition 3.5 An IFS $\mathcal{F}$ is said to be topologically contractive on a compact set $K \subset X$ if $F(K) \subset K^{\circ}$.

Theorem 3.6 (1) An affine IFS $\mathcal{F}$ has a attractor if and only $F$ is topologically contractive on some convex body.

(2) A Möbius IFS $\mathcal{F}$ on $\widehat{\mathbb{C}}$ has an attractor $A \neq \widehat{\mathbb{C}}$ if and only if $F$ is topologically contractive on some nonempty proper compact subset of $\widehat{\mathbb{C}}$.

(3) A projective IFS $\mathcal{F}$ has an attractor that avoids some hyperplane if and only if $F$ is topologically contractive on the union of a nonempty finite set of disjoint convex bodies.

\section{Chaos game}

Although condition (2) in Definition 2.2 can be used as the basis of an algorithm to draw arbitrarily accurate approximations to an attractor of an IFS, such a method is inefficient because the number of computations grows exponentially with $k$. Also, it is necessary to have, a priori, an initial set $S$ that lies in the basin of the attractor, to initiate the iterative construction. More efficient is the chaos game algorithm, in which the attractor is approximated by a chaos game orbit. This has the benefits of low memory usage and more freedom in the choice of an initial set of the form $S=\left\{x_{0}\right\}$.

Throughout this paper, the following notation is used. Let $[N]:=\{1,2, \ldots, N\}$, and let $\Omega:=[N]^{\infty}$ denote the set of infinite strings on the alphabet $[N]$.

Definition 4.1 Given an IFS $\mathcal{F}=\left\{X ; f_{1}, f_{2}, \ldots, f_{N}\right\}$ and an $\omega \in \Omega$, the chaos game orbit of a point $x_{0} \in X$ with respect to $\omega$ is the sequence $\left(x_{n}\right)_{n=0}^{\infty}$, where

$$
x_{n}:=f_{\omega_{n}}\left(x_{n-1}\right),
$$


for $n=1,2, \ldots$ The chaos game orbit $\left(x_{n}\right)_{n=0}^{\infty}$ is called a random orbit of $x_{0}$ if there is $p \in(0,1 / N]$ such that, for each $k \in\{1,2, \ldots\}, \omega_{k}$ is selected randomly from $\{1,2, \ldots, N\}$ with the probability that $\omega_{k}=n$ being greater than or equal to $p$, regardless of the preceding outcomes, for all $n \in\{1,2, \ldots, N\}$. (In terms of conditional probability, $P\left(\omega_{k}=n \mid x_{0}, \omega_{1}, \omega_{2}, \ldots \omega_{k-1}\right)>p$. $)$ We say that a chaos game orbit $\left(x_{n}\right)_{n=0}^{\infty}$ yields $A$ if

$$
A=\lim _{K \rightarrow \infty}\left\{x_{n}: n \geq K\right\}=\bigcap_{K \geq 1} \overline{\left\{x_{n}: n \geq K\right\}},
$$

where the limit is with respect to the Hausdorff metric.

An instructive example is provided by the IFS $\mathcal{F}=\left\{S^{1} ; f_{1}, f_{2}\right\}$ where $S^{1}$ is a circle, $f_{1}$ is an irrational rotation and $f_{2}$ is the identity. This IFS has unique attractor $A=S^{1}$ with basin $S^{1}$. Let $x_{0} \in S^{1}$, and compute a chaos game orbit by tossing a coin at each step. As $n$ gets large the orbit approaches $S^{1}$ with probability one.

Theorems 4.2 and 4.3 below tell us how chaos game orbits reveal attractors under very general conditions. It is at first sight surprising that it was successfully used, for example, to calculate images of attractors of non-contractive IFSs such as the one in Fig. 7. Indeed, there is no contractivity assumption in either Theorem 4.2 or 4.3. The proof of Theorem 4.2 appears in [33]. A metric space is proper if every closed ball is compact. Note that neither Definition 4.1 nor the following theorems say anything about convergence rates.

Theorem 4.2 Let $X$ be a proper complete metric space and $\mathcal{F}=\left\{X ; f_{1}, f_{2}, \ldots, f_{N}\right\}$ an IFS with attractor $A$ and basin $B$. If $\left(x_{n}\right)_{n=0}^{\infty}$ is a random orbit of $x_{0} \in B$ under $\mathcal{F}$, then with probability one this random orbit yields $A$.

A deterministic version of Theorem 4.2 appears in [29,31] and is stated as Theorem 4.3. A string $\sigma \in \Omega$ is disjunctive if every finite string is a substring of $\sigma$. By a substring of $\sigma$ we mean a string of the form $\sigma_{l} \sigma_{l+1} \cdots \sigma_{k}$ for integers $1 \leq l \leq k$. In fact, if $\sigma$ is disjunctive, then every finite string appears as a substring of $\sigma$ infinitely many times. For example, the binary Champernowne sequence $0100011011000001 \cdots$, formed by concatenating all finite binary strings in lexicographic order, is disjunctive. There are infinitely many disjunctive sequences if $N \geq 2$. Moreover, the set of disjunctive sequences is large in the sense that its complement in the natural metric space $\left(\Omega, d_{\Omega}\right)$ defined on $\Omega$ is a meager set and even a $\sigma$-porous set. The metric space $\left(\Omega, d_{\Omega}\right)$ is defined formally at the beginning of Section 6. A meager set is a set of the first Baire category; the definition of $\sigma$-porous can be found in $[93,127]$ and results using porosity in fractal geometry in $[46,83]$. The definition of a strongly-fibred attractor is given in Sect. 6, following Theorem 6.2.

Theorem 4.3 Let $X$ be a complete metric space and $F=\left\{X ; f_{1}, f_{2}, \ldots, f_{N}\right\}$ an IFS with strongly-fibred attractor $A$ and basin $B$. If $x_{0} \in B$ and $\left(x_{n}\right)_{n=0}^{\infty}$ is a chaos game orbit of $x_{0}$ with respect to a disjunctive sequence, then this chaos game orbit yields $A$.

Under certain additional conditions (see [29]), the initial point $x_{0}$ of the chaos game orbit is not restricted to lie in the basin of $A$. There is a set $D$ whose complement is $\sigma$-porous and if $x_{0} \in D$, then the conclusion of Theorem 4.3 remains true. 


\section{Phase transition}

Affine IFSs are basic to deterministic fractal geometry, perhaps because the first approximation to a non-linear map is affine, and because affine transformations, after similitudes, form a relatively simple group. Here we consider a family of affine IFSs depending on a parameter. The existence or non-existence of an attractor depends on the value of the parameter. As pointed out in Sect. 3, affine IFSs behave nicely in some ways; in particular, an affine IFS $\mathcal{F}$ has a unique attractor if and only if $\mathcal{F}$ is contractive. But there is an equivalent necessary and sufficient condition for $\mathcal{F}$ to possess an attractor, a condition involving the positive real parameter $\rho(\mathcal{F})$ called the joint spectral radius of $\mathcal{F}$. A dramatic geometric transition occurs at the precise value of $\rho(\mathcal{F})$ where the IFS changes from having an attractor to not having an attractor.

\subsection{Joint spectral radius}

The joint spectral radius of a set $\mathbb{L}=\left\{L_{i}, i \in I\right\}$ of linear maps on $\mathbb{R}^{n}$ was introduced by Rota and Strang [107], and the generalized spectral radius was introduced by Daubechies and Lagarias [49]. Berger and Wang [41] proved that the two concepts coincide for bounded sets of linear maps. A set of linear maps is bounded if there is an upper bound on their norms. (Note that all norms are equivalent, in the sense that there are positive constants $a$, b such that $a\|x\|_{1} \leq\|x\|_{2} \leq b\|x\|_{1}$ for all $x \in \mathbb{R}^{n}$.) The index set $I$ may be infinite, but it is assumed throughout this section that $\mathbb{L}$ is compact (as a subset of $\mathbb{R}^{n \times n}$ ). In particular, $\mathbb{L}$ compact implies that $\mathbb{L}$ is bounded. What follows is the definition of the joint spectral radius and the generalized spectral radius of $\mathbb{L}$. Let $\Omega_{k}$ be the set of all words $\sigma_{1} \sigma_{2} \cdots \sigma_{k}$, of length $k$, where $\sigma_{i} \in I, 1 \leq i \leq k$. For $\sigma=\sigma_{1} \sigma_{2} \cdots \sigma_{k} \in \Omega_{k}$, define

$$
L_{\sigma}:=L_{\sigma_{1}} \circ L_{\sigma_{2}} \circ \cdots \circ L_{\sigma_{k}}
$$

The generalized spectral radius is a generalization of the spectral radius $\rho(L)$ of a single linear map $L$, i.e. the maximum of the moduli of the eigenvalues of $L$.

Definition 5.1 For any set $\mathbb{L}$ of linear maps and any norm, the joint spectral radius of $\mathbb{L}$ is

$$
\widehat{\rho}=\widehat{\rho}(\mathbb{L}):=\limsup _{k \rightarrow \infty} \widehat{\rho}_{k}^{1 / k} \quad \text { where } \quad \widehat{\rho}_{k}:=\sup _{\sigma \in \Omega_{k}}\left\|L_{\sigma}\right\| .
$$

The generalized spectral radius of $\mathbb{L}$ is

$$
\rho=\rho(\mathbb{L}):=\limsup _{k \rightarrow \infty} \rho_{k}^{1 / k} \quad \text { where } \quad \rho_{k}:=\sup _{\sigma \in \Omega_{k}} \rho\left(L_{\sigma}\right)
$$

The following are well known properties of the joint and generalized spectral radii. 
(1) The joint spectral radius is independent of the particular norm.

(2) For an IFS consisting of a single linear map $L$, the generalized spectral radius is the ordinary spectral radius of $L$, the maximum of the moduli of the eigenvalues of $L$.

(3) For any real $\alpha>0$ we have $\rho(\alpha \mathbb{L})=\alpha \rho(\mathbb{L})$ and $\widehat{\rho}(\alpha \mathbb{L})=\alpha \widehat{\rho}(\mathbb{L})$.

(4) If $\mathbb{L}$ is bounded, then the joint spectral radius equals the generalized spectral radius.

If $\mathcal{F}$ is an affine IFS, infinite or not, the joint spectral radius is defined as the joint spectral radius of the set of linear parts of the functions in $\mathcal{F}$.

Definition 5.2 A set $\left\{L_{i}, i \in I\right\}$ of linear maps is called reducible if these linear maps have a common nontrivial invariant subspace. The set is irreducible if it is not reducible. An affine IFS is reducible (irreducible) if the set of linear parts is reducible (irreducible).

In terms of matrices, a set of linear maps is reducible if and only if there exists an invertible matrix $T$ such that all $L_{i}$ can be simultaneously put in a block uppertriangular form:

$$
T^{-1} L_{i} T=\left(\begin{array}{ll}
A_{i} & * \\
0 & B_{i}
\end{array}\right),
$$

for all $i$, with $A_{i}$ and $B_{i}$ square, and $*$ is any matrix with suitable dimensions. The joint spectral radius $\rho(\mathcal{F})$ is equal to $\max \left(\rho\left(\left\{A_{i}\right\}\right), \rho\left(\left\{B_{i}\right\}\right)\right)$.

Definition 5.3 An IFS, consisting of finitely or infinitely many affine functions, is compact if the set of $(n+1) \times n$ matrices that represent the functions is compact as a subset $\mathbb{R}^{(n+1) \times n}$.

The following theorem is proved in [32].

Theorem 5.4 A compact affine IFS $\mathcal{F}$ on $\mathbb{R}^{n}$ has an attractor if and only if $\rho(\mathcal{F})<1$. Moreover, if $\rho(\mathcal{F})<1$, then the attractor is unique and the basin is $\mathbb{R}^{n}$; if $\rho(\mathcal{F})>1$ and $\mathcal{F}$ is irreducible, then there does not exist a nonempty bounded set $A$ such that $F(A)=A$.

\subsection{Transition}

Theorem 5.4 states that $\rho(\mathcal{F})=1$ is the transition value between $\mathcal{F}$ having and not having an attractor. This transition is especially dramatic in the case of a linear IFS. Let $\mathcal{F}=\left\{\mathbb{R}^{n} ; L_{i}, i \in I\right\}$ be a linear IFS, an IFS all of whose functions are linear. In this case, if $\rho(\mathcal{F})<1$, then $\mathcal{F}$ has a unique attractor $A=\{0\}$, the origin. We say, in this case, that the attractor is trivial. If, on the other hand, $\rho(\mathcal{F})>1$, then by Theorem 5.4, the IFS $\mathcal{F}$ has no attractor, in fact no invariant set, i.e., no set $A$ such that $F(A)=A$. What is surprising is that, at the transition value $\rho(\mathcal{F})=1$ between the trivial invariant set and no invariant set, there is a geometric "blowup", a non-trivial invariant set. This is the content of the following theorem [32]. 
Fig. 5 An eigenset of Example 5.8 (color figure online)

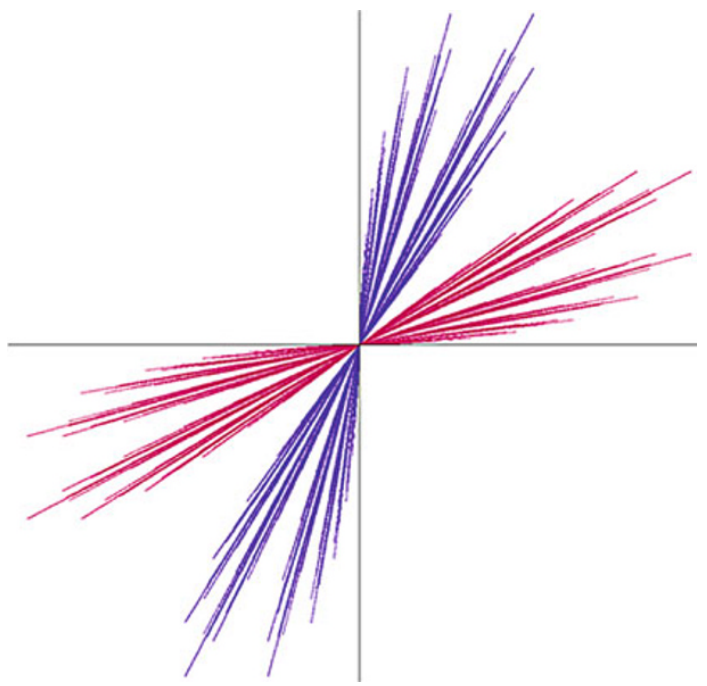

Fig. 6 An eigenset of Example 5.9 (color figure online)

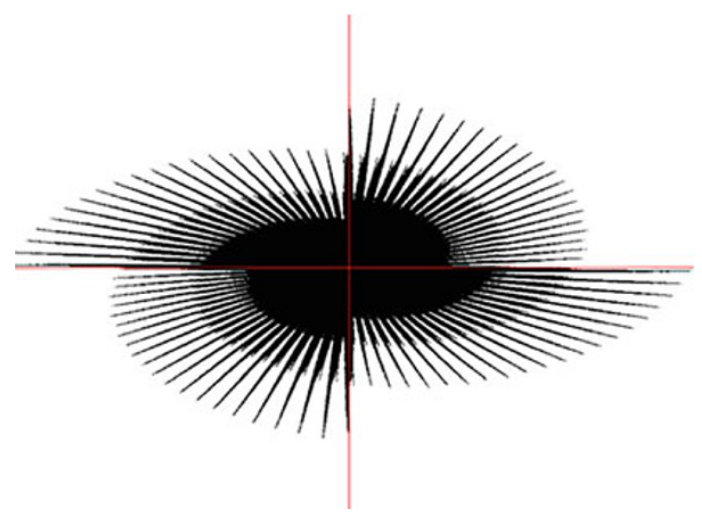

Theorem 5.5 A compact, irreducible, linear IFS $\mathcal{F}$ with $\rho(\mathcal{F})=1$ has a compact invariant set that is centrally symmetric, star-shaped, and full dimensional.

A set $S$ is centrally symmetric if $-x \in S$ whenever $x \in S$. A set $S$ is star-shaped if $\lambda x \in S$ for all $x \in S$ and all $0 \leq \lambda \leq 1$. And a set $S$ is full-dimensional if the affine hull of $S$ is $\mathbb{R}^{n}$. Figures 5 and 6 show two examples of the transition phenomenon at $\rho(\mathcal{F})=1$, transitioning between trivial invariant set and no invariant set. These two examples are further detailed below.

The theory outlined above can be reformulated as an analog to the eigenvalue problem in linear algebra.

Definition 5.6 Let $\mathcal{F}$ be an IFS and consider the eigen-equation

$$
F(Y)=\lambda Y,
$$


where $F$ is the usual Hutchinson operator of $\mathcal{F}, \lambda \in \mathbb{R}, \lambda>0$, and $Y \neq\{0\}$ is a compact set in Euclidean space. The value $\lambda$ in Eq. 5.1 above will be called an eigenvalue of $\mathcal{F}$, and $Y$ a corresponding eigenset.

When $\mathcal{F}$ consists of a single linear function $L$, an eigenvalue-eigenvector pair $(\lambda, x)$ of $L$ satisfies the eigen-equation. Even for a single linear function, however, there are more interesting eigenvalue-eigenset pairs. For example, let $L: \mathbb{R}^{2} \rightarrow \mathbb{R}^{2}$ be a linear map with no nontrivial invariant subspace, equivalently no real eigenvalue. Although $L$ has no real eigenvalue, $L$ does have an eigen-ellipse, an ellipse $E$, centered at the origin, such that $L(E)=\lambda E$, for some real $\lambda>0$. Although easy to prove, the existence of an eigen-ellipse is not universally known.

If Eq. 5.1 is rewritten as

$$
\left(\frac{1}{\lambda} \mathcal{F}\right) Y=Y
$$

then an eigenset for $F$ is an invariant set of $\frac{1}{\lambda} F$, and Theorem 5.5 can be formulated as follows.

Theorem 5.7 A compact, irreducible, linear IFS $\mathcal{F}$ has exactly one eigenvalue which is equal to the joint spectral radius $\rho(\mathcal{F})$ of $\mathcal{F}$. There is a corresponding eigenset that is centrally symmetric, star-shaped, and full dimensional.

Example 5.8 Fig. 5 shows an eigenset for the IFS $\mathcal{F}=\left\{\mathbb{R}^{2} ; L_{1}, L_{2}\right\}$, where

$$
L_{1}=\left(\begin{array}{ll}
10 & 10 \\
8 & 0
\end{array}\right), \quad L_{2}=\left(\begin{array}{ll}
8 & 0 \\
10 & 10
\end{array}\right)
$$

The eigenvalue appears to be $5+\sqrt{105}$, the value of the largest eigenvalue of $L_{1}$. The part of the set shown in red is, to viewing accuracy, the image of the whole set under $L_{1}$. The part of the set shown in blue is, similarly, the image of the whole set under $L_{2}$. The coordinate axes are indicated in black.

Example 5.9 Figure 6 shows an eigenset for the IFS $\mathcal{F}=\left\{\mathbb{R}^{2} ; L_{1}, L_{2}\right\}$, where

$$
L_{1}=\left(\begin{array}{ll}
0.02 & 0 \\
0 & 1
\end{array}\right), \quad L_{2}\left(\begin{array}{ll}
0.0594 & -1.98 \\
0.495 & 0.01547
\end{array}\right)
$$

The eigenvalue is 1 . The coordinate axes are indicated in red.

Theorem 5.7 cannot be extended to affine IFSs. It is true that, for a compact, irreducible, affine IFS, a real number $\lambda>0$ is an eigenvalue if $\lambda>\rho(\mathcal{F})$ and is not an eigenvalue if $\lambda<\rho(\mathcal{F})$. However, if $\mathcal{F}$ is not linear, there are examples where $\rho(\mathcal{F})$ is an eigenvalue and examples where it is not. 


\section{Fibres and addresses}

\subsection{Coordinate maps and coding maps}

This section concerns the assignment of addresses to the points in an attractor of an IFS $\mathcal{F}$. In the notation of Sect. 4 , the set $\Omega$ is called the code space for an IFS $\mathcal{F}$ consisting of $N$ functions. For a string $\omega \in \Omega$, denote the $n^{\text {th }}$ element in the string by $\omega_{n}$, and denote by $\omega \mid n$ the string consisting of the first $n$ symbols in $\omega$, i.e., $\omega \mid n=\omega_{1} \omega_{2}, \cdots \omega_{n}$. The code space $\Omega$ is a compact metric space with distance function $d_{\Omega}$ defined by

$$
d_{\Omega}(\omega, \sigma)= \begin{cases}0 & \text { if } \omega=\sigma, \\ 2^{-k} & \text { where } k \text { is the least positive integer such that } \omega_{k} \neq \sigma_{k} .\end{cases}
$$

The space $\left(\Omega, d_{\Omega}\right)$ is homeomorphic to the classical Cantor set treated as a subset of the unit interval with the usual topology.

Let $A$ be an attractor of $\mathcal{F}$ on a complete metric space $X$. To each $\omega \in \Omega$ we associate a fibre $A_{\omega} \subset A$ according to

$$
A_{\omega}:=\bigcap_{k \in \mathbb{N}} f_{\omega \mid k}(A) \in H(A)
$$

where

$$
f_{\omega \mid k}:=f_{\omega_{1}} \circ f_{\omega_{2}} \circ \cdots \circ f_{\omega_{k}}
$$

We have [71, Proposition 4.3.2]

$$
A=\bigcup_{\omega \in \Omega} A_{\omega}
$$

Following Kieninger [71, p.89, Definition 4.2.7], define the coordinate map $\pi_{\mathcal{F}}$ : $\Omega \rightarrow H(A)$ for $A$ (w.r.t. the IFS $\mathcal{F}$ ) by

$$
\pi_{\mathcal{F}}(\omega)=A_{\omega}
$$

for all $\omega \in \Omega$. The set of addresses of a point $x \in A$ is defined to be

$$
\pi_{\mathcal{F}}^{-1}(\{x\}):=\left\{\omega \in \Omega: x \in \pi_{\mathcal{F}}(\omega)\right\} .
$$

A point $x$ may have many addresses and many points may have the same address. To describe addresses, we use the notation $\overline{\sigma_{1} \sigma_{2} \cdots \sigma_{m}}$ to mean the periodic point $\sigma_{1} \sigma_{2} \cdots \sigma_{m} \sigma_{1} \cdots \sigma_{m} \sigma_{1} \cdots$. For example, $\overline{12}=12121 \ldots$ We also use the notation, when $\theta \in[N]^{k}$ and $\rho \in \Omega$,

$$
\theta \rho=\theta_{1} \theta_{2} \cdots \theta_{k} \rho_{1} \rho_{2} \rho_{3} \cdots=\theta_{1} \theta_{2} \cdots \theta_{k} \rho=\theta \rho_{1} \rho_{2} \rho_{3} \cdots
$$




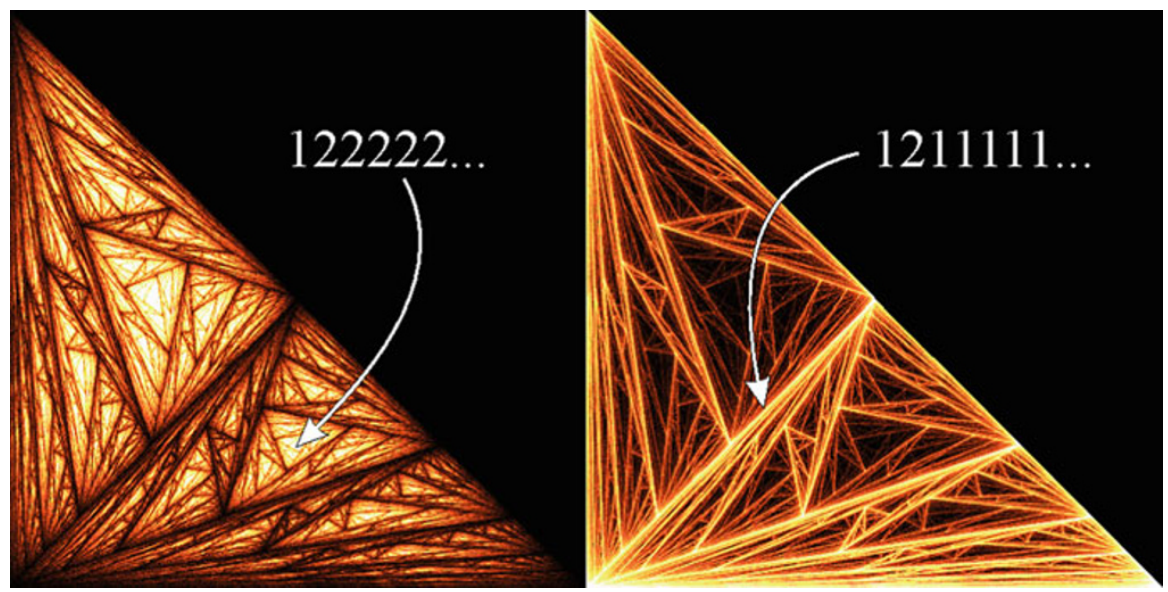

Fig. 7 Fibres of an attractor of a strongly fibered IFS consisting of two affine maps acting on a triangular subset of $\mathbb{R}^{2}$ (color figure online)

The notions of fibres and addresses are illustrated in Fig. 7, using the IFS

$$
\mathcal{F}=\left\{\boldsymbol{\Lambda} ; f_{1}(x, y)=(x+y / 2, y / 2), f_{2}(x, y)=(y / 2,-x-y / 2+1)\right\}
$$

where $\boldsymbol{\Delta} \subset \mathbb{R}^{2}$ is the filled triangle with vertices $(0,0),(1,0),(0,1)$. The IFS $(6.1)$ possesses a unique attractor $A=\boldsymbol{\Delta}$, but there is no metric on $\boldsymbol{\Delta}$ generating the standard topology and such that the IFS (6.1) is contractive. Some of the fibres are line segments and others are points. For example, $A_{\overline{1}}=\left\{(x, 0) \in \mathbb{R}^{2}: 0 \leq x \leq 1\right\}$ and $A_{\overline{2}}$ is the point with coordinates $(0.25,0.5)$. In order to illustrate some of the fibres of $A$ (w.r.t. $\mathcal{F}$ ) we have rendered $A$ in two different ways in Fig. 7; the image on the left uses bright colours to emphasize fibres which are points while the image on the right emphasizes fibres which are line segments. In the figure, an address of a fibre that is point (on the left) and an address of a fibre that is a line segment (on the right) are given.

Definition 6.1 If $\pi_{\mathcal{F}}(\omega)$ is a singleton for all $\omega \in \Omega$, then $A$ is said to be point-fibred (w.r.t. $\mathcal{F}$ ).

When $A$ is point-fibred, by slight abuse of notation, we treat the coordinate map $\pi_{\mathcal{F}}$ as a mapping

$$
\pi_{\mathcal{F}}: \Omega \rightarrow A
$$

In this case

$$
\pi_{\mathcal{F}}(\sigma)=\lim _{k \rightarrow \infty} f_{\sigma \mid k}\left(x_{0}\right)
$$

for $x_{0} \in B$, where $B$ is the basin of $A$. The following statement is an immediate consequence of [65, Section 3.1] and the definition of point-fibred. 
Theorem 6.2 If $\mathcal{F}$ is contractive, then its attractor is point-fibred.

A point belonging to the attractor of a point-fibred IFS may have one or many addresses, but there is only one point with any given address. If, for every open set $V \subset X$ such that $V \cap A \neq \emptyset$ there is a fibre $A_{\omega} \subset V$, then $A$ is said to be stronglyfibred. Figure 7 illustrates a strongly-fibred attractor that is not point-fibred. We have

$$
\text { point-fibred } \Longrightarrow \text { strongly-fibred. }
$$

An example of an IFS with a unique attractor that is not strongly-fibred is

$$
\left\{X ; f_{1}(\theta)=\theta, f_{2}(\theta)=\theta+\alpha\right\}
$$

where $X$ is a circle, $\theta \in X$ is the point with angular coordinate $\theta$, and $2 \pi / \alpha$ is irrational: the attractor is $A=X$ and $A_{\omega}=A$ for all $\omega \in \Omega$.

The coordinate map interacts with the shift map on code space. The shift map $S: \Omega \rightarrow \Omega$ is defined by $S\left(\omega_{1} \omega_{2} \omega_{3} \cdots\right)=\omega_{2} \omega_{3} \cdots$, and the inverse shift map $S_{n}: \Omega \rightarrow \Omega$ is defined by $S_{n}(\omega)=n \omega$ for $n \in[N]$. Both $S$ and $S_{n}$ are continuous. A subset $W \subset \Omega$ is called shift invariant if $S(W) \subseteq W$.

The following theorem is a simplified version of some results of Kieninger [71, Lemma 4.6.6 and Propositions 4.2.10 and 4.3.22].

Theorem 6.3 Let $A$ be an attractor of an IFS $\mathcal{F}$ on a complete metric space $X$. The coordinate map $\pi_{\mathcal{F}}: \Omega \rightarrow H(A) \subset H(X)$ is upper semicontinuous as a mapping from the metric space $\left(\Omega, d_{\Omega}\right)$ to the metric space $\left(H(X), d_{H(X)}\right)$. The following diagram commutes for all $n \in[N]$.

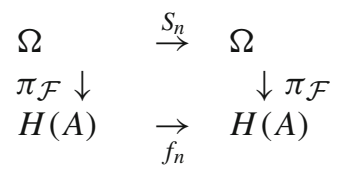

If $\mathcal{F}$ is point-fibred, then $\pi_{\mathcal{F}}: \Omega \rightarrow A$ is continuous.

This leads to the following definition.

Definition 6.4 Given an IFS $\mathcal{F}$ on a complete metric space $X$, a coding map for $\mathcal{F}$ is a continuous surjection $\varphi: \Omega \rightarrow K \subset X$ such that the following diagram commutes for each $n \in[N]$.

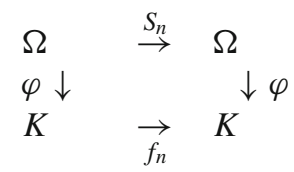

In this case $K$ is called a topological self-similar set. 
If $A$ is a point-fibred attractor for $\mathcal{F}$ then $\varphi=\pi_{\mathcal{F}}$ is a coding map for $\mathcal{F}$ with $K=A$. A point-fibred attractor is a topological self-similar set. In Definition 6.4 the nomenclature "topologically self-similar set" comes from the work of Kigami [73] and Kameyama [69], mainly in the context of analysis on fractals; it refers to the readily proved fact that $K$ is invariant for $F$, i.e. $K=\cup f_{n}(K)=F(K)$.

Definition 6.4 leads to Kameyama's Fundamental Question [69]: Can a topological self-similar set be associated with a contractive IFS? This question can be reformulated: if $\varphi: \Omega \rightarrow K \subset X$ is a coding map for an IFS $\mathcal{F}$ on a complete metric space $X$, then is $\mathcal{F}$, restricted to $K$, contractive?

To prove that the answer to his question is "no", Kameyama constructs an IFS $\mathcal{F}$ where $N=2$ and the metric space $X$ is defined abstractly in terms of the code space $\Omega$. Kameyama's IFS is non-geometrical, and his question remains open when $X$ and the transformations are geometrically simple, as described in the introduction. But for affine IFSs the answer is "yes". Indeed, it is quite surprising that for an affine IFS, contractivity is assured solely by the existence of a coding map [4].

Theorem 6.5 If $\mathcal{F}=\left\{\mathbb{R}^{n} ; f_{1}, f_{2}, \ldots f_{N}\right\}$ is an affine IFS with a coding map $\varphi$ : $\Omega \rightarrow K \subset \mathbb{R}^{n}$, then $\mathcal{F}$ is contractive when restricted to the affine hull of $\varphi(\Omega)$. In particular, if $\varphi(\Omega)$ contains a non-empty open subset of $\mathbb{R}^{n}$, then $\mathcal{F}$ is contractive on $\mathbb{R}^{n}$.

The affine hull of a subset $S$ of $\mathbb{R}^{n}$ is the smallest affine subspace containing $S$.

\subsection{Sections of coordinate maps}

If $\mathcal{F}$ is point-fibred, then the coordinate map $\pi_{\mathcal{F}}: \Omega \rightarrow A$ assigns a point in the attractor $A$ of $\mathcal{F}$ to each infinite string. It would be convenient if, for an IFS $\mathcal{F}$ with point fibred attractor $A$, we had a map in the direction opposite to that of the coordinate map, assigning to each point $A$ a string in $\Omega$. Since the coordinate map $\pi_{\mathcal{F}}: \Omega \rightarrow A$ need not be injective, the following definition is useful, for example in the construction of fractal transformations, discussed in Sect. 9.

Definition 6.6 A section of a coordinate map $\pi_{\mathcal{F}}: \Omega \rightarrow A$ is a map $\tau: A \rightarrow \Omega$ such that $\pi_{\mathcal{F}} \circ \tau$ is the identity. For $x \in A$, the string $\tau(x)$ is referred to as the address of $x$ with respect to the section $\tau$. The set $\Omega_{\tau}:=\tau(A)$ is called the address space of the section $\tau$. The section $\tau$ is called shift invariant when $\Omega_{\tau}$ is shift invariant.

Properties of sections of coordinate maps are discussed in [21, Section 3].

Example 6.7 Let $F=\left\{\mathbb{R} ; f_{0}, f_{1}\right\}$ where $f_{0}(x)=\frac{1}{2} x$ and $f_{1}(x)=\frac{1}{2} x+\frac{1}{2}$. In this case the IFS possesses a unique attractor, the real interval $[0,1]$. For $\omega=\omega_{1} \omega_{2} \cdots$, the "binary decimal expansion map" $\pi(\omega):=\sum_{k=1}^{\infty} \omega_{k} 2^{-k}$ is a coding map. Note that $\pi(1000 \cdots)=\frac{1}{2}=\pi(0111 \cdots)$. There are two shift invariant section maps. One is the map that sends each point of $[0,1]$ to its binary decimal expansion that contains no infinite string of consecutive zeros. The other is the map that sends each point of $[0,1]$ to its binary expansion that contains no infinite string of consecutive ones. 


\section{Classification of attractors}

\subsection{Set of overlap}

In this section the attractor $A$ of an IFS $\mathcal{F}=\left\{X ; f_{1}, f_{2}, \ldots, f_{N}\right\}$ is classified according to how the sets $f_{n}(A), n=1,2, \ldots, N$, intersect. Throughout we assume that the functions $f_{n}$ are pairwise distinct when restricted to an attractor under discussion.

Definition 7.1 Let $A$ be an attractor of an IFS $\mathcal{F}$. The set of overlap of $A$ (w.r.t. $\mathcal{F}$ ) is

$$
O=O(A):=\left\{x \in A: f_{i}(A) \cap f_{j}(A) \neq \emptyset \text {, some } i \neq j, i, j \in[N]\right\}
$$

(a) $A$ is non-overlapping or $\operatorname{disjoint}($ w.r.t. $\mathcal{F}$ ) if $O(A)=\emptyset$;

(b) $A$ is overlapping (w.r.t. $\mathcal{F}$ ) if $O(A) \neq \emptyset$;

(c) $A$ is slightly overlapping or just-touching (w.r.t. $\mathcal{F}$ ) if it is not disjoint and the interior of $O$ (w.r.t. the subspace topology on $A$ ) is empty;

(d) $A$ is strongly overlapping (w.r.t. $\mathcal{F}$ ) if it is overlapping but not slightly overlapping, i.e. if the interior of $O$ (w.r.t the subspace topology on $A$ ) is nonempty;

(e) $A$ obeys the open set condition (w.r.t. $\mathcal{F}$ ) if there is a nonempty open set $U$ in the basin of $A$ such that $F(U) \subset U$ and $f_{i}(U) \cap f_{j}(U)=\emptyset$ whenever $i \neq j$, $i, j \in[N]$;

(f) $A$ is post-critically finite (w.r.t. $\mathcal{F}$ ) if it is connected, $\mathcal{F}$ is a contractive similitude IFS, and $\cup_{n \geq 1} S^{n}\left(\pi_{\mathcal{F}}^{-1}(O(A))\right.$ is a finite set;

(g) $A$ is finitely ramified if $O(A)$ comprises finitely many distinct points.

When the meaning is clear from the context we omit the caveat "w.r.t. $\mathcal{F}$ ". The terminology in Definition 7.1 mainly follows Kieninger [71] and Kigami [72,73]. Note that Strichartz [117] uses a less general definition of post-critically finite.

Next, some properties of attractors with each of the properties in Definition 7.1, and some relationships between these properties, are briefly outlined. For ease of exposition, it is assumed that $\mathcal{F}$ is contractive.

If $A$ is disjoint, then it is totally disconnected. If $A$ is a disjoint attractor of an injective IFS with $N \geq 2$, then it is perfect and it is homeomorphic to the classical Cantor set (where the relative topologies are understood). In this case the coordinate map $\pi_{\mathcal{F}}: \Omega \rightarrow A$ is a homeomorphism. The green spiral Cantor set in Fig. 3 illustrates a totally disconnected attractor (of an inverse IFS).

If $A$ obeys the open set condition, then it is just-touching. The converse, however, is not true [71, Remark 5.3.2]. The open set condition, together with invariant measures associated with the IFS, are used in the analysis of the Hausdorff-Besicovitch dimension $\mathcal{D}(A)$ of an attractor $A \subset \mathbb{R}^{M}$. Assume that $A$ is an attractor that obeys the open set condition w.r.t. a similitude IFS $\mathcal{F}$ on $\mathbb{R}^{M}$, with $\left\|f_{n}\left(x_{1}\right)-f_{n}\left(x_{2}\right)\right\|=s_{n}\left\|x_{1}-x_{2}\right\|$ for all $x_{1}, x_{2} \in \mathbb{R}^{M}$, where $s_{n}>0$ for all $n \in[N]$. Then (see for example [56, Theorem 9.3])

$$
\sum_{n=1}^{N} s_{n}^{\mathcal{D}(\mathcal{A})}=1
$$


Bandt and Rao [11] have recently discussed the relationship between the open set condition and the size of the set of overlap. They provide an example of a similitude IFS in $\mathbb{R}^{2}$ whose attractor $A$ is a Cantor set for which $\mathcal{D}(A)$ is arbitrarily small, but such that $A$ does not obey the open set condition. Various refinements and modifications of the open set condition are used to yield additional information about dimensions of attractors; see for example [102] and the references therein.

The topic of post-critically finite (p.c.f.) attractors is specialized, but central to analysis on fractals. Analysis on fractals involves the construction of Laplacians [73], harmonic analysis, Brownian motion [12], heat kernels [77] (see the review in [74]), and differential equations on fractals [117]. See also Hambly [61] and Teplyaev [118]. The attractor of the standard Sierpinski IFS

$$
\left\{\mathbb{R}^{2} ;(x / 2, y / 2),(x / 2+1 / 4, y / 2+\sqrt{3} / 4),(x / 2+1 / 2, y / 2)\right\}
$$

is an example of a p.c.f. fractal. We note that the structures related to analysis on fractals, such as random walks, invariant measures, Dirichlet kernels, and so on, can be described in terms of analysis on equivalence classes on the underlying code space, or in terms of analysis on limit sets of sequences of combinatorial graphs.

Recently, the relationship between the open set condition, the property of being p.c.f., the property of being finitely ramified, and other separation conditions, have been explored in the general setting of graph-directed IFSs [83]; see [51,96].

Strongly overlapping point-fibred systems are related to $\beta$-transformations [100], Bernoulli convolutions, and coding theory, areas which attract current attention; see for example [7,64]. They are also related to fractal transformations as discussed in Sect. 9.

\subsection{Kneading invariant}

The topology of a point-fibred attractor is determined by the address structure of its critical set, a set related to the set of overlap. The definition of the critical set and the related address structure, called the kneading invariant, are defined below. The theory of kneading invariants developed from the study of the orbit of the critical point under an iterated unimodal map, by Collet and Eckman [47], in connection with dynamical systems and chaotic dynamics, and later substantially extended by Milnor and Thurston [86].

Bandt and Keller [9] initiated a systematic study of the topology of contractive IFS attractors based on the code space structure of the set of overlap. Here we adapt the more recent development by Kameyama [69]. If $S$ is a set with finitely many distinct elements then $\# S$ is the number of distinct elements in $S$; if $S$ has infinitely many distinct elements then $\# S=\infty$.

Definition 7.2 Let $A$ be a point-fibred attractor of an IFS $\mathcal{F}$. We say that

$$
\left.C=O(A) \cup\left(\bigcup_{n \in[N]}\left\{x: \#\left\{y \in A: f_{n}(y)=x\right\} \geq 2\right\}\right\}\right)
$$


is the critical set of $A$ (w.r.t. $\mathcal{F}$ ), and

$$
\mathcal{A}=\left\{\pi_{\mathcal{F}}^{-1}(c): c \in C\right\} \subset 2^{\Omega}
$$

is the kneading invariant of $A$ (w.r.t. $\mathcal{F}$ ).

From the continuity of the coordinate map $\pi_{\mathcal{F}}: \Omega \rightarrow A$ in the point-fibred case, it follows that a point-fibred attractor $A$ is homeomorphic to the quotient space $\Omega / \sim$ where $\sigma \sim \theta$ if $\pi_{\mathcal{F}}(\sigma)=\pi_{\mathcal{F}}(\theta)$. Conversely, the equivalence relation derived from a set $\mathcal{A} \subset \Omega$ determines an "IFS" $\mathcal{F}=\left\{X ; f_{1}, f_{2}, \ldots, f_{N}\right\}$, but the space $X$ may not be a Hausdorff space and hence not metrizable. (If it is Hausdorff then it is metrizable with a metric constructed using the coding map.) Theorem 7.3 below tells us how the structure of $\Omega / \sim$ is determined by the structure of the kneading invariant $\mathcal{A}$. It does so by characterizing the addresses of a point $x$ such that $\# \pi_{\mathcal{F}}^{-1}(x) \geq 2$. We use the following notation: if $\theta \in[N]^{k}$ for some $k \in\{1,2, \ldots\}$ and $\Theta \subset[N]^{\infty}$, then $\theta \Theta:=\left\{\omega \in[N]^{\infty}: \omega \mid k=\theta\right.$ and $\left.S^{k} \omega \in \Theta\right\}$, and $\emptyset \Theta:=\Theta$.

Theorem 7.3 Let A be a point-fibred attractor of an IFS $\mathcal{F}$, and let $\mathcal{A}$ be the kneading invariant of $A$ w.r.t. $\mathcal{F}$.

(i) If $x \in A$ and $\# \pi_{\mathcal{F}}^{-1}(x) \geq 2$, then there uniquely exists $\Theta \in \mathcal{A}$ and $\theta \in[N]^{k} \cup \emptyset$ for some $k \in\{1,2, .$.$\} such that \pi_{\mathcal{F}}(\theta \Theta)=\{x\}$.

(ii) If $\Theta, \Xi \in \mathcal{A}, \theta \in[N]^{k} \cup \emptyset$ for some $k \in\{1,2, .$.$\} , and \theta \Theta \cap \Xi \neq \emptyset$, then $\theta \Theta \subset \Xi$. Moreover if $\theta \Theta=\Xi$, then $\Theta=\Xi$ and $\theta=\emptyset$.

Theorem 7.4, a simplified version of a theorem of Kameyama [69], provides sufficient conditions under which the kneading invariant determines a canonical quotient space which is homeomorphic to the given attractor.

Theorem 7.4 Conversely to Theorem 7.3, suppose that $\mathcal{A} \subset 2^{\Omega}$ has the property (ii) in Theorem 7.3 and $\# \Theta \geq 2$ for each $\Theta \in \mathcal{A}$. Define an equivalence relation $\sim$ on $\Omega$ by $\sigma \sim \rho$ if either $\sigma=\rho$ or $\sigma, \rho \in \theta \Theta$ for some $\Theta \in \mathcal{A}$, for some $\theta \in[N]^{k} \cup \emptyset$ for some $k \in\{1,2, \ldots\}$. Let $A$ be the quotient space $\Omega / \sim$ and let $\pi: \Omega \rightarrow A$ be the projection. Then there exist continuous maps $f_{n}: A \rightarrow A$ for $n=1,2, \ldots, N$, such that the diagram (6.3), with $\varphi:=\pi$, commutes. If the critical set $C$ is finite and $\pi_{\mathcal{F}}^{-1}(x)$ is compact for each $x \in A$, then $\left\{A ; f_{1}, f_{2}, \ldots, f_{N}\right\}$ is an IFS with point-fibred attractor $A$ and kneading invariant $\mathcal{A}$.

Theorem 7.4 can be used to construct homeomorphisms between suitable pairs of point-fibred attractors whose kneading invariants are the same. For example, let attractors $A_{1}$ and $A_{2}$ of injective IFSs $\mathcal{F}_{1}$ and $\mathcal{F}_{2}$ obey the conditions of Theorems 7.3 and 7.4, i.e., assume that the attractors are point-fibred, that the critical sets are finite, and that $\left(\pi_{\mathcal{F}_{i}}\right)^{-1}(x)$ is compact for every $x \in A_{i}, i=1,2$, where $\pi_{\mathcal{F}_{1}}$ and $\pi_{\mathcal{F}_{2}}$ are their respective coordinate maps. Let $\tau_{\mathcal{F}_{1}}$ be a section of $A_{1}$ (w.r.t. $\mathcal{F}_{1}$ ) and let $\tau_{\mathcal{F}_{2}}$ be a section for $A_{2}$ (w.r.t. $\mathcal{F}_{2}$ ), and assume that the critical sets are both finite, and that the kneading invariants are the same, say $\mathcal{A}_{1}=\mathcal{A}_{2}$. Then, similarly to $[17$, Theorem 
1], it follows from Theorem 7.4 that a homeomorphism $T: A_{1} \rightarrow A_{2}$ is defined by

$$
\begin{aligned}
T & =\pi_{\mathcal{F}_{2}} \circ \tau_{\mathcal{F}_{1}}, \\
T^{-1} & =\pi_{\mathcal{F}_{1}} \circ \tau_{\mathcal{F}_{2}} .
\end{aligned}
$$

Transformations between attractors, including some of this kind, are discussed in Sect. 9.

An example of a homeomorphic pair of tree-like IFS attractors is illustrated in Fig. 8. The tree-like set in black in the top image represents the Julia set $J_{\lambda}$ for the complex quadratic map $z \mapsto z^{2}+\lambda$ where $\lambda=-0.228157+i(1.115143)$. This Julia set is the attractor of the IFS

$$
\mathcal{J}_{\lambda}=\left\{\mathbb{Q} \subset \mathbb{C}: f_{1}(z)=\sqrt{z-\lambda}, f_{2}(z)=-\sqrt{z-\lambda}\right\}
$$

where $\mathbb{Q}$ is any compact set such that $f_{i}(\mathbb{Q}) \subset \mathbb{Q}$ for $i=1,2$, and the two branches $\pm \sqrt{z-\lambda}$ of the inverse of $z \mapsto z^{2}+\lambda$ are continuous on $\mathbb{Q}$. For an explanation of how such a Julia set can be viewed as the attractor of a suitable IFS, see [19]. The bottom image in Fig. 8 is the attractor of the similitude IFS $\left\{\mathbb{C}: f_{1}(z)=s \exp (i \theta) z, f_{2}(z)=\right.$

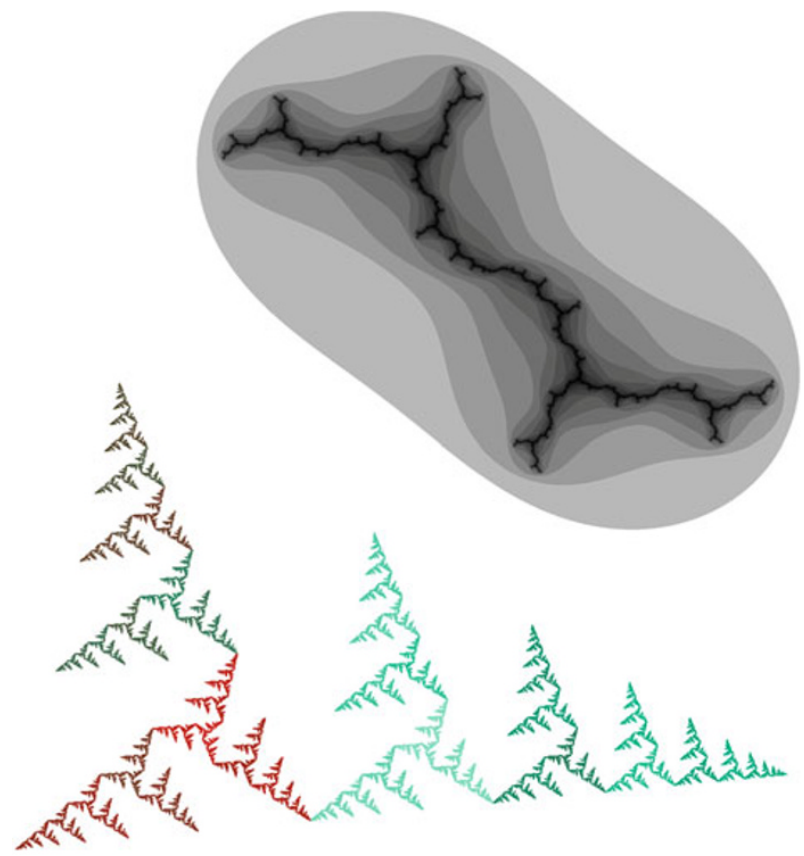

Fig. 8 Two tree-like sets, one a Julia set and the other the attractor of a similitude IFS, are homeomorphic because they have the same kneading invariant, discussed in Sect. 7.2. These two sets correspond to boundary points of associated Mandelbrot sets; see Sect. 8. Julia sets for a wide range of values of $\lambda$ can be calculated on the fly using inexpensive computer applications, for example [66] (color figure online) 
$s \exp (i \theta) z+1\}$ where $\theta=108 \pi / 180$ and $s=(\sqrt{5}-1) / 2$. The kneading invariant for each attractor (w.r.t. its IFS) is $\{111 \overline{2}, 211 \overline{2}\}$; see [10].

\section{Mandelbrot set for pairs of linear maps}

Given a family $\mathcal{F}_{\lambda}$ of contractive IFSs, each consisting of a pair of similitudes that depend on a single parameter $\lambda$, the set of points $\mathcal{M}$ in parameter space such that the attractor of $\mathcal{F}_{\lambda}$ is connected has emerged over the last twenty years as an area of interest. The set $\mathcal{M}$, called a Mandelbrot set, is the topic of this section.

If $\mathcal{F}$ is a contractive IFS with attractor $A$, then $A$ is connected if and only if, for any $r_{0} \leq r_{m} \leq N$, there exists a sequence $\left\{r_{1}, r_{2}, \ldots, r_{m-1}\right\} \subset\{1,2, \ldots, N\}$ such that $f_{r_{i}}(A) \cap f_{r_{i+1}}(A) \neq \varnothing$ for any $0 \leq i \leq m-1$. Also, if $A$ is connected, then it is locally connected; see for example [73, Proposition 1.6.4].

For the case $N=2$, there is a fundamental dichotomy for attractors: the attractor is connected if and only if

$$
f_{1}(A) \cap f_{2}(A) \neq \emptyset
$$

and is otherwise totally disconnected. This leads to the notion of Mandelbrot sets for parameterized families of pairs of transformations. Let $A_{\lambda}$ denote the attractor of the contractive IFS

$$
\mathcal{F}_{\lambda}=\left\{\mathbb{C}: f_{1}(z)=\lambda z, f_{2}(z)=\lambda z+1\right\}
$$

for $\lambda \in \mathbb{D}:=\{z \in \mathbb{C}:|z|<1\}$. The set

$$
\mathcal{M}:=\left\{\lambda \in \mathbb{D}: A_{\lambda} \text { is connected }\right\}
$$

was introduced in [24] as an analog to the classical Mandelbrot set $\mathcal{M}_{1}$. Indeed, it is shown in [10] that attractors $A_{\lambda}$ of $\mathcal{F}_{\lambda}$ corresponding to some values of $\lambda$ that lie in the boundary of $\mathcal{M}$ are homeomorphic to Julia sets $J_{\tilde{\lambda}}$ for $z \mapsto z^{2}+\tilde{\lambda}$, where the corresponding values of $\tilde{\lambda}$ belong to the boundary of $\mathcal{M}_{1}$. Such a pair of conjugate attractors is illustrated in Fig. 8. The set $\mathcal{M}$ has connections to Bernoulli convolutions, Dirichlet forms on fractals, and zeros of polynomials with integer coefficients. See for example [6,9,39,45,110-112,114].

To see the relationship between zeros of polynomials with integer coefficients and $\mathcal{M}$, note that finite compositions $f_{\sigma \mid k}(z)$ of the maps in $\mathcal{F}_{\lambda}$ give rise to polynomials of the form $f_{\sigma \mid k}(z)=(\lambda z)^{k}+\sum_{l=0}^{k-1}\left(\sigma_{l+1}-1\right) \lambda^{l}$, where each coefficient $\left(\sigma_{l+1}-1\right)$ is 0 or 1 . It follows that $\pi_{\mathcal{F}_{\lambda}}(\sigma)=\sum_{l=0}^{\infty}\left(\sigma_{l+1}-1\right) \lambda^{l}$ for all $\lambda \in \mathbb{D}$. It, in turn, follows from the remark containing equation (8.1), that the attractor $A_{\lambda}$ of $\mathcal{F}_{\lambda}$ is connected, for given $\lambda \in \mathbb{D}$, if and only if there exists $\sigma, \tau \in[2]^{\infty}$ with $\sigma \neq \tau$, such that

$$
\sum_{l=0}^{\infty}\left(\sigma_{l+1}-1\right) \lambda^{l}=\sum_{l=0}^{\infty}\left(\tau_{l+1}-1\right) \lambda^{l} .
$$



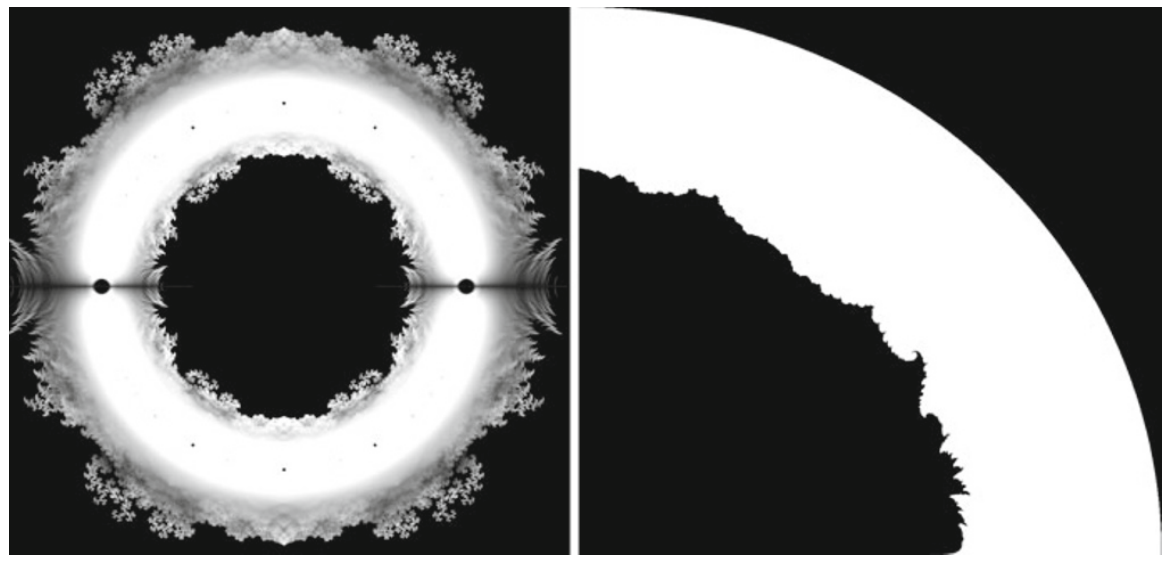

Fig. 9 Left density of zeros, in the complex plane, for all polynomials of degree 28 with each coefficient equal to +1 or -1 . The origin of coordinates is at the center of the image. The point with coordinate $(1,0)$ lies in the center of the small black disk to the right of the center. Right a rough plot of the Mandelbrot set (in white) with $\operatorname{Re} \lambda>0$ and $\operatorname{Im} \lambda>0$, for the IFS (8). Both images relate to the Mandelbrot set $\mathcal{M}$ for a similitude IFS, see text (color figure online)

Since all such sequences of zeros and ones are permissible, the attractor is connected if and only if $\lambda$ is a solution of equation (8.2) for some distinct pair of such sequences. The above implies

$$
A_{\lambda} \text { is connected } \Longleftrightarrow \sum_{l=0}^{\infty} \gamma_{l+1} \lambda^{l}=0 \text { with } \gamma_{l} \in\{0, \pm 1\} \text { and not all } \gamma_{l}=0 \text {. }
$$

On the left in Fig. 9 we illustrate the density of zeros in shades of gray, where black means no zeros/pixel, in the complex plane, for all polynomials of degree 28, with each coefficient equal to +1 or -1 . Since we do not include coefficients (other than the leading one) equal to 0 , the points which are not black, which lie inside the circle $|\lambda|=1$, lie in $\mathcal{M}$. (The centers of the black disks lie on the circle $|\lambda|=1$ ). On the right in Fig. 9 we show a rough plot of $\mathcal{M} \cap\{\lambda \in \mathbb{D}: \operatorname{Re} \lambda>0, \operatorname{Im} \lambda>0\}$ in white. This was computed by "brute force" - compute a digital approximation to $A$ and then see whether or not (digitized $\left.f_{1}(A)\right) \cap\left(\right.$ digitized $\left.f_{2}(A)\right)=\emptyset$. The common features, and the differences, of the two images, the one on the right and the one on the left, are striking.

The observation (8.3) was made by Bousch [45], who used it to show that $\mathcal{M}$ is both connected and locally connected. (Douady and Hubbard [54] proved the connectivity of the classical Mandelbrot set $\mathcal{M}_{1}$. Their conjecture, made in 1982, that $\mathcal{M}_{1}$ is locally connected, remains open.) The equivalence (8.3) was also used by Bandt [6] as the basis of a fast algorithm for the computation of high resolution pictures of $\mathcal{M}$. This revealed surprising features and led to interesting questions and conjectures concerning the geometry and topology of $\mathcal{M}$. For example, Bandt noted that the complement of $\mathcal{M}$ splits into many components and that $\mathcal{M}$ is not the closure of its 
interior; and he conjectured that $\mathcal{M} \backslash \mathbb{R}$ is the closure of its interior. Bandt also noted a relationship between $\mathcal{M}$ and Bernoulli convolutions, reviewed in [103]. Bandt's ideas were further developed by Solomyak and Xu [114], who made progress on Bandt's conjecture and studied properties of complex analogues of Bernoulli convolutions. Loci of connectedness of attractors of IFSs continues to be an active area of research; see for example [82].

\section{Fractal transformations}

While properties of classical geometrical transformations (e.g. affine, conformal, projective) are well known, much less is known about their rough counterparts, fractal transformations. Fractal transformations were introduced in $2005[15,16]$, and they have applications to image synthesis [97], photography [57], and digital imaging [21]. A fractal transformation takes the attractor of one IFS to the attractor of another IFS. Figures 10 and 11, explained in more detail later in this section, provide visualizations of fractal transformations.

A fractal transformation is defined in terms of a coordinate map, discussed in Sect. 6.1, and a section of a coordinate map, discussed in Sect. 6.2. Here it is assumed that all IFSs are point-fibred so that each possesses a coordinate map, as given by
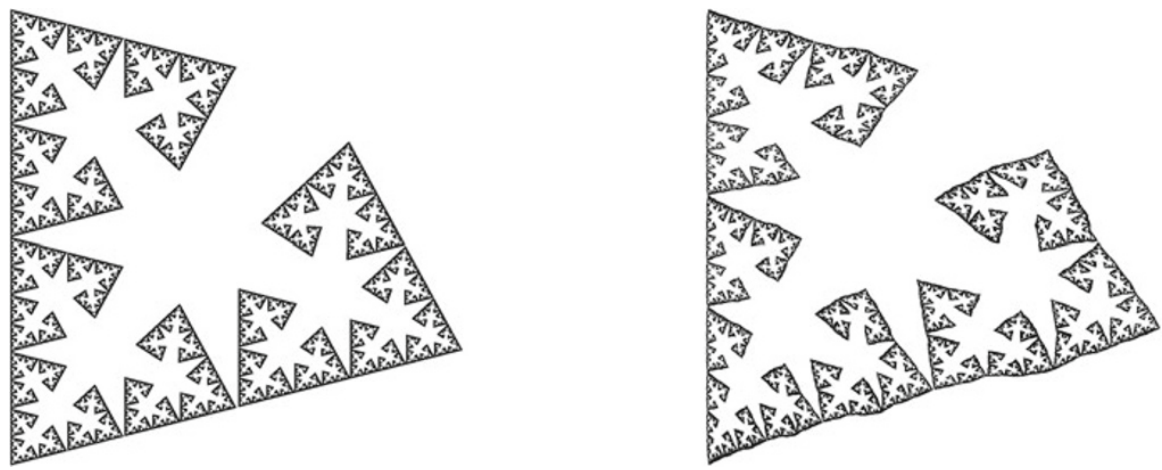

Fig. 10 Fractal homeomorphism: attractor on the left, attractor fractally transformed on the right
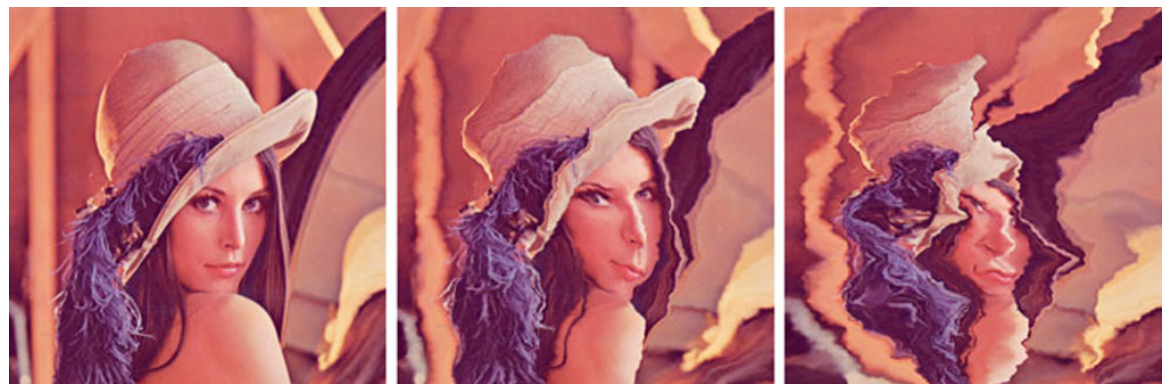

Fig. 11 Two fractal homeomorphisms applied to the original picture (color figure online) 
Eq. 6.2. Given two point-fibred IFSs $\mathcal{F}$ and $\mathcal{G}$ with respective attractors $A_{\mathcal{F}}$ and $A_{\mathcal{G}}$, a fractal transformation is basically a function $h: A_{\mathcal{F}} \rightarrow A_{\mathcal{G}}$ that sends a point in $A_{\mathcal{F}}$ to the point in $A_{\mathcal{G}}$ with the same address. More precisely, assume that $\mathcal{F}$ and $\mathcal{G}$ have the same number of functions, and let $\pi_{\mathcal{F}}$ and $\pi_{\mathcal{G}}$ be the respective coordinate maps.

Definition 9.1 A transformation $h: A_{\mathcal{F}} \rightarrow A_{\mathcal{G}}$ is called a fractal transformation if

$$
h=\pi_{\mathcal{G}} \circ \tau_{\mathcal{F}}
$$

for some shift invariant section $\tau_{\mathcal{F}}$ of $\mathcal{F}$. If $h$ is a homeomorphism, then $h$ is called a fractal homeomorphism.

Proposition 9.2 If a fractal transformation $h=\pi_{\mathcal{G}} \circ \tau_{\mathcal{F}}: A_{\mathcal{F}} \rightarrow A_{\mathcal{G}}$ is a homeomorphism, then there exists a shift invariant section $\tau_{\mathcal{G}}$ of $\pi_{\mathcal{G}}$ such that the following diagram commutes:

$$
\begin{array}{ll}
A_{\mathcal{F}} & \underset{h}{ } A_{\mathcal{G}} \\
\tau_{\mathcal{F}} \searrow & \swarrow \tau_{\mathcal{G}}
\end{array}
$$

Conversely, if there exists sections $\tau_{\mathcal{F}}$ and $\tau_{\mathcal{G}}$ and a homeomorphism $h$ such that the above diagram commutes, then $h=\pi_{\mathcal{G}} \circ \tau_{\mathcal{F}}$ and $h^{-1}=\pi_{\mathcal{F}} \circ \tau_{\mathcal{G}}$.

The commuting diagram means that the homeomorphism $h$ takes each point $x \in A_{\mathcal{F}}$ with address $\omega:=\tau_{\mathcal{F}}(x)$ to the point $y \in A_{\mathcal{G}}$ with the same address $\omega=\tau_{\mathcal{G}}(y)$. Two basic questions are (1) given an IFS, how to construct a shift invariant section, and (2) when is a fractal transformation a homeomorphism.

Definition 9.3 An IFS $\mathcal{F}=\left\{X ; f_{1}, f_{2}, \ldots, f_{N}\right\}$ is said to be injective if the map $f_{i}: X \rightarrow X$ is injective, for $i=1,2, \ldots, N$.

We consider the first question in the case that $\mathcal{F}$ is an injective IFS.

Definition 9.4 For an IFS $\mathcal{F}$ with attractor $A$, a mask is a partition $M=\left\{M_{i}, 1 \leq\right.$ $i \leq N\}$ of $A$ such that $M_{i} \subseteq f_{i}(A)$ for all $f_{i} \in \mathcal{F}$. Given an injective IFS $\mathcal{F}$ and a mask $M$, consider the function $T: A \rightarrow A$ defined by

$$
T(x):=f_{i}^{-1}(x)
$$

when $x \in M_{i}$. The itinerary $\tau_{M}(x)$ of a point $x \in A$ is the string $\omega_{0} \omega_{1} \omega_{2} \cdots \in \Omega$, where $\omega_{k}$ is the unique integer $1 \leq i_{k} \leq N$ such that

$$
T^{k}(x) \in M_{\omega_{k}} .
$$


Example 9.5 A particular mask for an IFS with $N$ functions and attractor $A$ is defined by $M_{1}=f_{1}(A)$ and

$$
M_{k}=f_{k}(A) \backslash \bigcup_{i=1}^{k-1} f_{i}(A),
$$

for $k=2,3, \ldots, N$. This is called the tops mask. There is a tops mask for every permutation of the functions, i.e., every permutation of $[N]$.

The following theorem provides an answer to the first question posed above; it states that any shift invariant section is constructed from a mask.

Theorem 9.6 Let $\mathcal{F}$ be a contractive and injective IFS.

(1) If $M$ is a mask, then $\tau_{M}$ is a shift invariant section of $\pi$.

(2) If $\tau$ is a shift invariant section of $\pi$, then $\tau=\tau_{M}$ for some mask $M$.

The section from a tops mask is given by

$$
\tau(x)=\max \pi^{-1}(x),
$$

where the maximum is with respect to the lexicographic order on $\Omega$.

Question (2) involves establishing conditions under which $\mathcal{F}$ and $\mathcal{G}$ have related kneading invariants; see the discussion following Theorem 7.4. In the following subsection we consider two geometrically simple types of IFS, for which interesting families of fractal transformations can be established.

\subsection{Bi-affine IFSs on $\mathbb{R}^{2}$}

A bi-affine function from $\mathbb{R}^{2}$ to $\mathbb{R}^{2}$ is more general than an affine function but less general than a quadratic function.

Definition 9.7 A function $f: \mathbb{R}^{2} \rightarrow \mathbb{R}^{2}$ is called bi-affine if it has the form

$$
f(x, y)=\mathbf{a}+\mathbf{b} x+\mathbf{c} y+\mathbf{d} x y,
$$

where bold face letters represent vectors in $\mathbb{R}^{2}$. Call a bi-affine function nondegenerate if $\mathbf{d} \neq \mathbf{0}$ and neither $\mathbf{b}$ nor $\mathbf{c}$ is a scalar multiple of $\mathbf{d}$. In particular, neither $\mathbf{b}$ nor $\mathbf{c}$ is the zero vector. A description of the geometric degeneracies that occur in these situations is described in [35]. An IFS $\mathcal{F}$ is called bi-affine if each function in $\mathcal{F}$ is a non-degenerate bi-affine function.

It is easy to verify that a function is bi-affine if and only if, for a fixed $x$ or a fixed $y$, it is affine in the other variable:

$$
\begin{aligned}
& f\left((1-\alpha) x_{1}+\alpha x_{2}, y\right)=(1-\alpha) f\left(x_{1}, y\right)+\alpha f\left(x_{2}, y\right) \quad \text { and } \\
& f\left(x,(1-\alpha) y_{1}+\alpha y_{2}\right)=(1-\alpha) f\left(x, y_{1}\right)+\alpha f\left(x, y_{2}\right)
\end{aligned}
$$


for all $x_{1}, x_{2}, y_{1}, y_{2}, \alpha \in \mathbb{R}$. Interpreted geometrically, these equations mean that

(1) horizontal and vertical lines are taken to lines, and

(2) proportions along horizontal and vertical lines are preserved.

This elementary class of functions, with connections to classic geometric results of Brianchon and Lambert dating back to the 18th century, proves extremely versatile for applications involving fractal transformations. A photography application [57], for example, is based on it. See also [18].

Note that the unique bi-affine function taking $(0,0),(1,0),(1,1),(0,1)$ to the points $\mathbf{p}_{0}, \mathbf{p}_{1}, \mathbf{p}_{2}, \mathbf{p}_{3}$, respectively, is

$$
f(x, y)=\mathbf{p}_{0}+\left(\mathbf{p}_{1}-\mathbf{p}_{0}\right) x+\left(\mathbf{p}_{3}-\mathbf{p}_{0}\right) y+\left(\mathbf{p}_{2}+\mathbf{p}_{0}-\mathbf{p}_{1}-\mathbf{p}_{3}\right) x y .
$$

Let $\square$ denote the unit square with vertices $(0,0),(1,0),(1,1),(0,1)$. The following Theorem provides sufficient conditions for a bi-affine function to be a contraction.

Theorem 9.8 Let $f(x, y)=\mathbf{p}_{0}+\left(\mathbf{p}_{1}-\mathbf{p}_{0}\right) x+\left(\mathbf{p}_{3}-\mathbf{p}_{0}\right) y+\left(\mathbf{p}_{2}+\mathbf{p}_{0}-\mathbf{p}_{1}-\mathbf{p}_{3}\right) x y$ be a non-degenerate bi-affine function such that $\mathbf{p}_{0} \mathbf{p}_{1} \mathbf{p}_{2} \mathbf{p}_{3}$ is a convex quadrilateral $P$. If there is an $s, 0 \leq s<1$, such that (1) each side of $P$ has length less than or equal to $s$, (2) each diagonal has length less than or equal to $\sqrt{2} s$, and (3) the vector sum of any two incident sides has length less or equal to $\sqrt{2} s$, then $f$ is a contraction on $\square$.

Attention is now restricted to the case of a non-degenerate bi-affine function taking $\square$ into itself. For such a bi-affine function, the conditions in Theorem 9.8 are not too restrictive. The intention is to form an IFS consisting of such functions, which must, according to Theorem 3.4, have a unique attractor. Given two such bi-affine IFSs with the same number of functions, fractal transformations, such as those illustrated in Fig. 11, can be produced as described below.

Consider a bi-affine IFS $\mathcal{F}=\left\{\square ; f_{1}, f_{2}, f_{3}, f_{4}\right\}$, where the four functions are determined by the images of the four vertices of $\square$ as shown in Fig. 12. Each of the four images $f_{i}(\square), i=1,2,3,4$, of the square contains exactly one vertex of $\square$. The attractor of $\mathcal{F}$ is $\square$ itself. For "most" choices of the center and side points, $\mathcal{F}$ satisfies the conditions of Theorem 9.8 and hence $\mathcal{F}$ is a contractive IFS.

Next let $M_{\mathcal{F}}=\left\{M_{1}, M_{2}, M_{3}, M_{4}\right\}$ be the tops mask for $\mathcal{F}$. Explicitly, $M_{1}$ is the closed quadrilateral $A T O Q, M_{2}$ is the open quadrilateral $O Q B R$ together with the segments $(Q, B],[B, R],[R, O), M_{3}$ is the open quadrilateral $O R C S$ together with the segments $(R, C],[C, S],[S, O)$, and $M_{4}$ is the open quadrilateral $O S D T$ together with the segments $(S, D],[D, T)$.

Now consider a second bi-affine IFS $\mathcal{G}=\left\{\square ; g_{1}, g_{2}, g_{3}, g_{4}\right\}$ of the same type with points $O^{\prime}, Q^{\prime}, R^{\prime}, S^{\prime}, T^{\prime}$ replacing $O, Q, R, S, T$, and with mask $M_{\mathcal{G}}$ defined exactly as it was for $M_{\mathcal{F}}$. The masks $M_{\mathcal{F}}$ and $M_{\mathcal{G}}$ induce shift invariant sections $\tau_{\mathcal{F}}$ and $\tau_{\mathcal{G}}$, respectively, as guaranteed by Theorem 9.6.

Theorem 9.9 For the pair of IFS described above, the maps $\pi_{\mathcal{G}} \circ \tau_{\mathcal{F}}$ and its inverse $\pi_{\mathcal{F}} \circ \tau_{\mathcal{G}}$ are homeomorphisms. 
Fig. 12 An IFS consists of four bi-affine functions. Points labeled with lower case letters are the images of points labeled with upper case letters. The attractor is the unit square (color figure online)

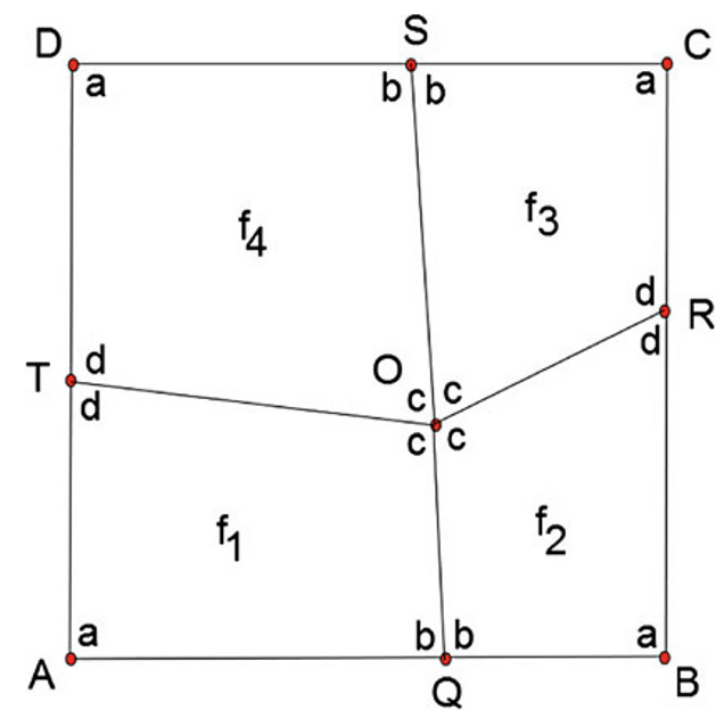

In this case the attractors of both IFSs are identical, the square. The fractal homeomorphism can be visualized as follows. Define an image as a function $c: \square \rightarrow \mathcal{C}$, where $\mathcal{C}$ denotes the color palette, for example $\mathcal{C}=\{0,1,2, \ldots, 255\}^{3}$. If $h$ is any homeomorphism from $\square$ onto $\square$, define the transformed image $h(c): \square \rightarrow \mathcal{C}$ by

$$
h(c):=c \circ h .
$$

When $h$ is a fractal homeomorphism as constructed via Theorem 9.9, we get a visualization of the fractal homeomorphism by looking at how an image is transformed. This is done, for example, in Fig. 11.

\subsection{Three dimensional tri-affine fractal homeomorphisms}

The preceding discussion generalizes to higher dimensions; see for example [23]. Diverse (multi-affine) fractal transformations can be constructed. Figures 13 and 14 illustrate an example.

\subsection{Special overlapping IFSs}

The bi-affine and tri-affine IFSs described above are non-overlapping, in the sense that if $A$ is the attractor of the IFS $\mathcal{F}$, then $(f(A) \cap g(A))^{o}=\emptyset$ for all distinct $f, g \in \mathcal{F}$. To determine whether a strongly overlapping IFS (in the sense of Definition 7.1d) fractal transformation is a homeomorphism is, in general, difficult. In this section a criterion is provided for a simple family of overlapping IFSs. 

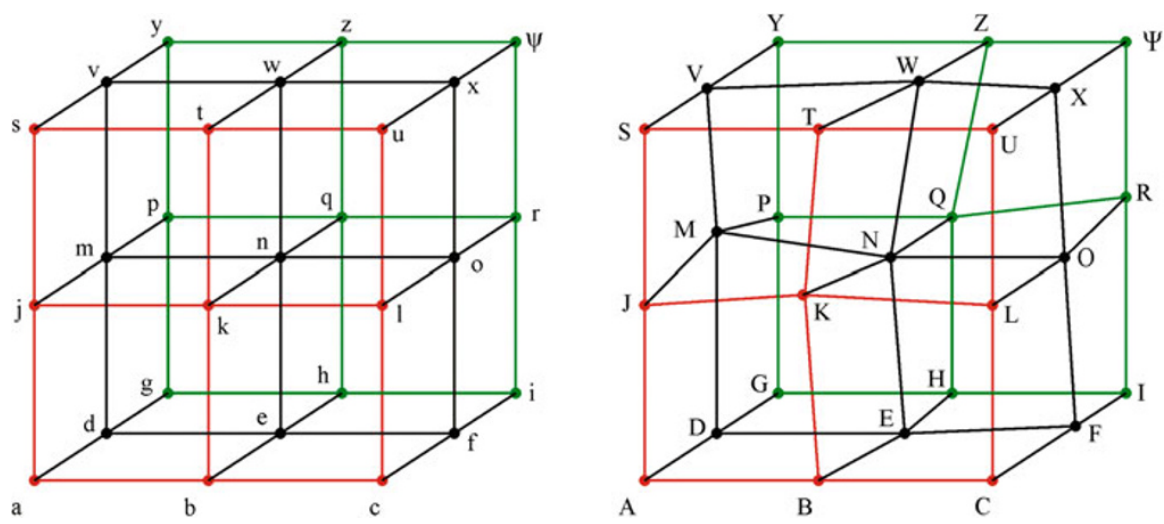

Fig. 13 Two labellings of points in the unit cube, used to define two tri-affine IFSs. The smaller cubes on the left represent a three-dimensional "tiling" of the cube by copies of itself. The corresponding objects on the right have ruled surfaces but fit together perfectly (color figure online)
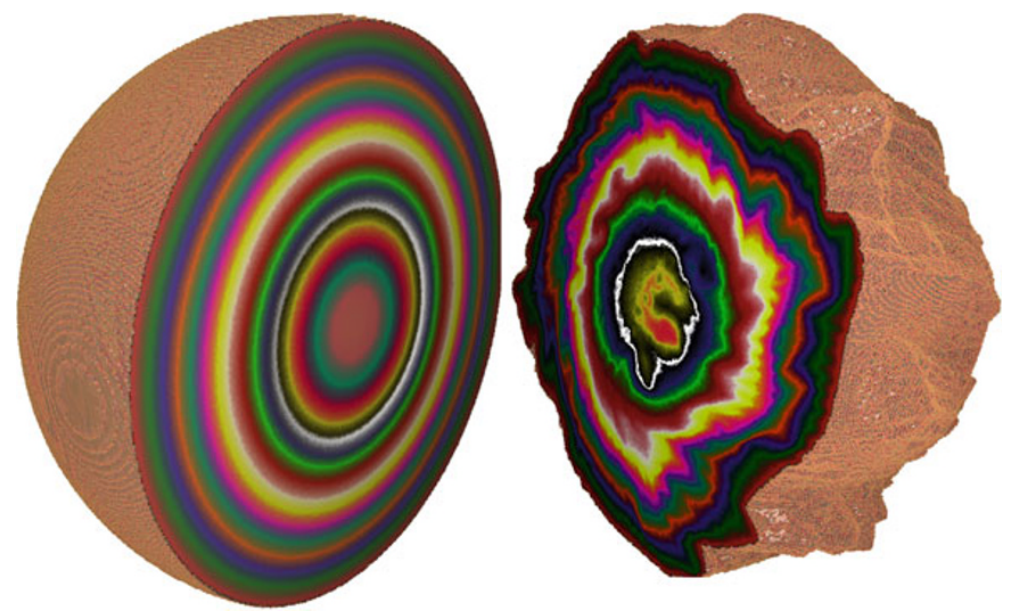

Fig. 14 This illustrates the action of a tri-affine fractal homeomorphism on a three-dimensional picture that resembles a gob-stopper, one of those candies that have multiple different colored layers that are revealed by sucking on the candy. The before and after images have been cut in half to reveal the effects of the transformation on the internal structure of the spherical picture. Digital technology used in connection with tomography can be used to explore such three dimensional pictures (color figure online)

\section{A special overlapping IFS is an IFS}

$$
\mathcal{F}=\left\{[0,1] ; f_{0}(x), f_{1}(x)\right\},
$$

on the unit interval, where the functions are continuous, increasing, contractions that satisfy

$$
f_{0}(0)=0, \quad f_{1}(1)=1, \quad 0<f_{1}(0)<f_{0}(1)<1,
$$


the last condition guaranteeing that $\mathcal{F}$ is strongly overlapping.

To construct a fractal transformation $h:[0,1] \rightarrow[0,1]$ from the attractor of one special overlapping IFS to the attractor of another, a section of the coordinate map must be constructed, which in turn requires designating a mask. A single point $q \in[0,1]$ determines a pair of related masks. The partitions are of the form

$$
M_{q}^{+}=\{[0, q),[q, 1]\} \quad \text { or } \quad M_{q}^{-}=\{[0, q],(q, 1]\},
$$

where $f_{1}(0)<q<f_{0}(1)$. The point $q$ will be called the mask point. For a masked special overlapping IFS, the two sections $\tau_{q}^{+}$and $\tau_{q}^{-}$, one corresponding to $M_{q}^{+}$and the other corresponding to $M_{q}^{-}$, are as follows: $\tau_{q}^{+}=\omega_{0} \omega_{1} \omega_{2} \cdots$ and $\tau_{q}^{-}=\sigma_{0} \sigma_{1} \sigma_{2} \cdots$, where

$$
\omega_{n}=\left\{\begin{array}{ll}
0 & \text { if }\left(T_{q}^{+}\right)^{n}(x)<q \\
1 & \text { if }\left(T_{q}^{+}\right)^{n}(x) \geq q
\end{array} \quad \text { and } \quad \sigma_{n}= \begin{cases}0 & \text { if }\left(T_{q}^{-}\right)^{n}(x) \leq q \\
1 & \text { if }\left(T_{q}^{-}\right)^{n}(x)>q\end{cases}\right.
$$

and where

$$
T_{q}^{+}(x)=\left\{\begin{array}{ll}
f_{0}^{-1 x} & \text { if } x<q \\
f_{1}^{-1 x} & \text { if } x \geq q
\end{array} \quad \text { and } \quad T_{q}^{-}(x)= \begin{cases}f_{0}^{-1 x} & \text { if } x \leq q \\
f_{1}^{-1 x} & \text { if } x>q .\end{cases}\right.
$$

For a special overlapping masked IFS $\mathcal{F}$, the itineraries

$$
\alpha_{q}:=\tau_{q}^{-}(q) \quad \text { and } \quad \beta_{q}:=\tau_{q}^{+}(q)
$$

of the mask point $q$ will be called the critical itineraries.

The following theorem [22] states that, for two special overlapping IFSs, whether or not a fractal transformation is a homeomorphism depends only on these two critical itineraries.

Theorem 9.10 Given two special overlapping masked IFSs $\mathcal{F}$ and $\mathcal{G}$ with respective mask points $q$ and p, coordinate maps $\pi_{\mathcal{F}}$ and $\pi_{\mathcal{G}}$, sections $\tau_{\mathcal{F}}^{ \pm}$and $\tau_{\mathcal{G}}^{ \pm}$, and critical itineraries $\alpha_{q}, \beta_{q}$ and $\alpha_{p}, \beta_{p}$, the fractal transformations $\pi_{\mathcal{G}} \circ \tau_{\mathcal{F}}^{ \pm}$and $\pi_{\mathcal{F}} \circ \tau_{\mathcal{G}}^{ \pm}$are homeomorphisms if and only if $\tau_{\mathcal{F}}^{+}(q)=\tau_{\mathcal{G}}^{+}(p)$ and $\tau_{\mathcal{F}}^{-}(q)=\tau_{\mathcal{G}}^{-}(p)$.

\section{IFS tiling}

Computer generated drawings of tilings of the plane by self-similar figures appear in papers beginning in the 1980s and 1990s, for example the lattice tiling of the plane by copies of the twindragon. Tilings constructed from an IFS often possess global symmetry and self-replicating properties. Research on such tilings include the work of Bandt, Gelbrich, Gröchenig, Hass, Kenyon, Lagarias, Madych, Radin, Solomyak, Strichartz, Thurston, Vince, and Wang and the more recent work of Akiyama and Lau; see for example [3, 5, 58-60,70,75,78, 104, 113, 116, 119, 122] and the list of references in [123]. Even aperiodic tilings can be put into the IFS context; see for example the fractal version of the Penrose tilings [8]. 
The use of the inverses of the IFS functions in the study of tilings is well-established, in particular in many of the references cited above. However, in this section we introduce a simple and yet unifying technique for constructing both the classical and new IFS tilings. Theorems 10.4 and 10.5 show that the resulting tiling very often covers the entire basin of the attractor. In the case of tilings of Euclidean space, the basin is the entire Euclidean space.

Throughout this section, $\mathcal{F}$ is a contractive IFS. By tile we simply mean a compact set. If $A$ is the attractor of an IFS $\mathcal{F}$, it is sometimes possible to tile the basin of $A$ with non-overlapping copies of $A$ or perhaps with non-overlapping tiles of several shapes related to $A$. The attractor $A$ of any IFS in this section will be just-touching and such that the interior $A^{o}$ is nonempty. Let $\mathcal{F}=\left\{\{X\} ; f_{1}, f_{2}, \ldots, f_{N}\right\}$ be an invertible IFS with just-touching attractor $A$. For any string $\theta \in \Omega$, a tiling $T_{\theta}=T_{\mathcal{F}, \theta}$ will be constructed as follows. Extending the notation $f_{\theta \mid k}=f_{\theta_{1}} \circ f_{\theta_{2}} \circ \cdots \circ f_{\theta_{k}}$ used in Section 6, let

$$
\left(f^{-1}\right)_{\theta \mid k}=f_{\theta_{1}}^{-1} \circ f_{\theta_{2}}^{-1} \circ \cdots \circ f_{\theta_{k}}^{-1} .
$$

Note that, in general, $\left(f^{-1}\right)_{\theta \mid k} \neq\left(f_{\theta \mid k}\right)^{-1}$. Given a positive integer $k$ and any $\omega \in$ $[N]^{k}$, let

$$
\begin{aligned}
t_{\theta, \omega} & =\left(\left(f^{-1}\right)_{\theta \mid k} \circ f_{\omega}\right)(A), \\
T_{\theta, k} & =\left\{t_{\theta, \omega}: \omega \in[N]^{k}\right\} .
\end{aligned}
$$

Since, for any $\omega \in[N]^{k}$, we have

$$
\left(f^{-1}\right)_{\theta \mid k} \circ f_{\omega}=\left(f^{-1}\right)_{\theta \mid k} \circ\left(f_{\theta_{k+1}}\right)^{-1} \circ f_{\theta_{k+1}} \circ f_{\omega}=\left(f^{-1}\right)_{\theta \mid k+1} \circ f_{\theta_{k+1} \omega},
$$

the inclusion

$$
T_{\theta} \subset T_{\theta, k+1}
$$

holds for all $k$. Therefore

$$
T_{\theta}=T_{\mathcal{F}, \theta}:=\bigcup_{k=1}^{\infty} T_{\theta, k}
$$

is a tiling of

$$
B(\theta):=\bigcup_{k=1}^{\infty}\left(f^{-1}\right)_{\theta \mid k}(A) \subseteq X,
$$

where the union is a nested union.

Under certain, not too restrictive conditions on $\theta$, conditions given in Theorem 10.4, $T_{\theta}$ tiles the entire basin of the attractor $A$. 
Example 10.1 The IFS $\mathcal{F}=\left\{\mathbb{R} ; f_{0}, f_{1}\right\}$ where $f_{1}(x)=x / 2$ and $f_{2}(x)=x / 2+1 / 2$ has attractor $[0,1]$. The tiling $T_{\mathcal{F}, \overline{1}}$ is the tiling of $[0, \infty)$ by unit intervals. The tiling $T_{\mathcal{F}, \overline{2}}$ is the tiling of $(-\infty, 1]$ by unit intervals. If $\theta \neq \overline{1}, \overline{2}$, then $T_{\mathcal{F}, \theta}$ tiles $\mathbb{R}$ by unit intervals.

Definition 10.2 For an invertible IFS $\mathcal{F}$ with attractor $A$, call $\theta \in \Omega$ full if there exists a compact set $A^{\prime} \subset A^{o}$ such that, for any positive integer $M$, there exist $n>m \geq M$ such that

$$
f_{\theta_{n}} \circ f_{\theta_{n-1}} \circ \cdots \circ f_{\theta_{m+1}}(A) \subset A^{\prime}
$$

Call $\theta \in \Omega$ reversible for IFS $\mathcal{F}$ if, for every positive integer $M$, there exists an $m \geq M$ such that

$$
\theta_{1} \theta_{2} \cdots \theta_{m-1} \theta_{m}=\omega_{m} \omega_{m-1} \cdots \omega_{2} \omega_{1} .
$$

where $\omega=\omega_{1} \omega_{2} \cdots \in \Omega$ is the address of some point in $A^{o}$. The following notation will be used: $\overleftarrow{\omega \mid m}:=\omega_{m} \omega_{m-1} \cdots \omega_{2} \omega_{1}$, so condition (10.1) can be expressed as $\theta \mid m=\overleftarrow{\omega \mid m}$.

A string $\sigma \in \Omega$ is periodic of period $p$ if $\sigma_{k+p}=\sigma_{k}$ for all $k=1,2, \ldots$ Recall from Sect. 4 that a string $\sigma \in \Omega$ is disjunctive if every finite word is a substring of $\sigma$. The set of disjunctive sequences is a large subset of $\Omega$ in a topological, in a measure theoretic, and in an information theoretic sense [115].

Proposition 10.3 Let $\mathcal{F}$ be an invertible IFS with attractor A with non-empty interior.

(1) There are infinitely many disjunctive strings in $\Omega$ if $N \geq 2$.

(2) Every disjunctive string is reversible.

(3) Every reversible string is full.

Theorem 10.4 Let $\mathcal{F}$ be a non-overlapping invertible IFS with attractor $A$ with nonempty interior and with basin $B$. If $\theta$ is full, then $T_{\mathcal{F}, \theta}$ covers $B$.

By the above proposition and theorem (which are proved in [38]), full strings are plentiful. In fact, according to the next result, also proved in [38], any string $\theta$ is full with probability 1 . Define a string $\theta \in \Omega$ to be a random string if it is chosen as follows: there is a $p \in(0,1 / N)$ such that, for each $k \in\{1,2, \ldots\}, \theta_{k}$ is selected randomly from $\{1,2, \ldots, N\}$, where the probability that $\sigma_{k}=n$ is greater than or equal to $p$, independently of $\theta_{1}, \theta_{2}, \ldots \theta_{k-1}$, for all $n \in[N]$. (See also Definition 4.1.)

Theorem 10.5 Let $\mathcal{F}=\left\{X ; f_{1}, f_{2}, \ldots, f_{N}\right\}$, where $X$ is compact, be an invertible IFS with just-touching attractor A with non-empty interior and with basin B. If $\theta \in \Omega$ is a random string, then, with probability 1 , the tiling $T_{\mathcal{F}, \theta}$ covers $B$.

Example 10.6 (Digit tiling of $\mathbb{R}^{n}$ ) The terminology "digit tiling"comes from the data used to construct the tiling, which is analogous to the usual base and digits used to represent the integers. An expanding matrix, i.e., linear function on $\mathbb{R}^{n}$, is an $n \times n$ matrix such that the modulus of each eigenvalue is greater than 1. Let $L$ be an expanding 
$n \times n$ integer matrix. A set $D=\left\{d_{1}, d_{2}, \ldots, d_{N}\right\}$ of coset representatives of the quotient $\mathbb{Z}^{n} / L\left(\mathbb{Z}^{n}\right)$ is called a digit set. It is assumed that $0 \in D$. By standard algebra results, for $D$ to be a digit set it is necessary that

$$
|D|=|\operatorname{det} L|
$$

Consider an affine IFS $\mathcal{F}:=\mathcal{F}(L, D)=\left\{\mathbb{R}^{n} ; f_{1}, f_{2}, \ldots, f_{N}\right\}$, where

$$
f_{i}(x)=L^{-1}\left(x-d_{i}\right) .
$$

Since $L$ is expanding, it is known that, with respect to a metric equivalent to the Euclidean metric, $L^{-1}$ is a contraction. Since $\mathcal{F}$ is contractive, there is a unique attractor $A$ called a digit tile. The basin of $A$ is all of $\mathbb{R}^{n}$. Note that a digit tile is completely determined by the pair $(L, D)$ and will be denoted $T(L, D)$. It is known [123] that a digit tile $T$ is the closure of its interior and its boundary has Lebesque measure 0 . The iterated function system $\mathcal{F}(L, D)$ is non-overlapping. If $\theta \in \Omega$ is full, then $T_{\mathcal{F}, \theta}$ is a tiling of $\mathbb{R}^{n}$ called a digit tiling. It is straightforward to show that, up to a rigid motion of $\mathbb{R}^{n}$, a digit tiling does not depend on $\theta$ as long as it is full. Under fairly mild assumptions [123, Theorem 4.3], a digit tiling is a tiling by translation by the integer lattice $\mathbb{Z}^{n}$ with the following self-replicating property: for any tile $t \in T_{L, D}$, it's image $L(t)$ is the union of tiles in $T_{L, D}$. For this reason, such a tiling is often referred to as a reptiling of $\mathbb{R}^{n}$.

Example 10.7 (Crystallographic tilings of $\mathbb{R}^{n}$ ) Gelbrich [58] generalized digit tiling from the lattice group $\mathbb{Z}^{n}$ to any crystallographic group $\Gamma$. Let $L: \mathbb{R}^{n} \rightarrow \mathbb{R}^{n}$ be an expanding linear map such that $L \Gamma L^{-1} \subset \Gamma$. If $D=\left\{d_{1}, \ldots, d_{N}\right\}$ is a set of right coset representatives of $\Gamma / L \Gamma L^{-1}$, then

$$
\mathcal{F}=\left\{\mathbb{R}^{n} ; L^{-1} d_{1}, \ldots, L^{-1} d_{N}\right\}
$$

is a contractive IFS with attractor $T(\Gamma, L, D)$ with non-empty interior, called a crystallographic tile. The Levy curve is an example of such a crystallographic tile (for the 2-dimensional crystallographic group $p 4$ ). A tiling $T_{\mathcal{F}, \theta}$ of $\mathbb{R}^{n}$ is called a crystallographic reptiling.

Example 10.8 (Chair tilings of $\mathbb{R}^{2}$ ) The IFS $\mathcal{F}=\left\{\mathbb{R}^{2} ; f_{1}, f_{2}, f_{3}, f_{4}\right\}$ where

$$
\begin{array}{ll}
f_{1}(x, y)=(x / 2, y / 2), & f_{3}(x, y)=(-x / 2+1, y / 2), \\
f_{2}(x, y)=(x / 2+1 / 4, y / 2+1 / 4), & f_{4}(x, y)=(x / 2,-y / 2+1),
\end{array}
$$

is an IFS whose attractor is a filled square with a missing quadrant, i.e., a chair shaped polygon. There are numerous other such polygonal tiles that are the attractors of just touching IFSs. For the chair tile, there are uncountably many tilings $T_{\mathcal{F}, \theta}$, i.e. uncountably many full strings $\theta$.

Example 10.9 (Triangular affine tilings of $\mathbb{R}^{2}$ ) Although we do not do so here, the significance of this tiling is that it can be used to extend a fractal homeomorphism 

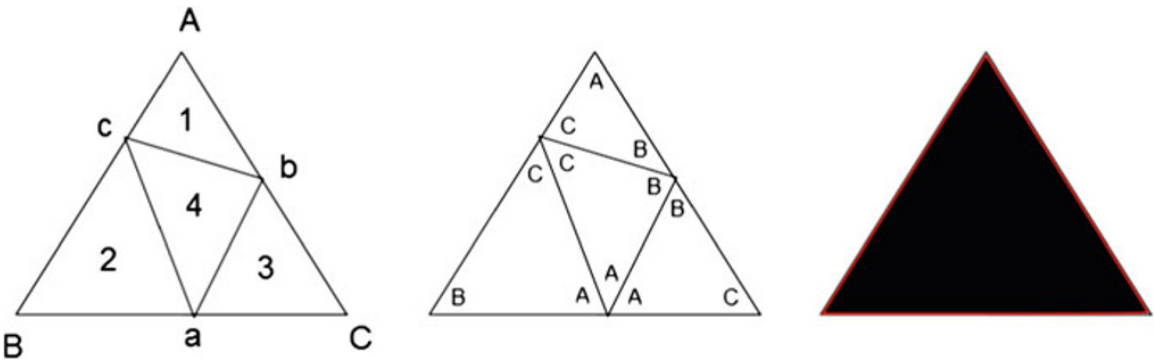

Fig. 15 (i) The points used to define the affine transformations of the IFS $F=\left\{\mathbb{R}^{2} ; f_{1}, f_{2}, f_{3}, f_{4}\right\}$; (ii) images of the triangle $A B C$; (iii) the attractor of the IFS $\left\{\mathbb{R}^{2} ; f_{1}, f_{2}, f_{3}, f_{4}\right\}$ (color figure online)

between two triangular attractors, to a fractal homeomorphism of the Euclidean plane. Consider the IFS $\mathcal{F}=\left\{\mathbb{R}^{2} ; f_{1}, f_{2}, f_{3}, f_{4}\right\}$ where each $f_{n}$ is an affine transformation defined as follows. Let $A, B$, and $C$ denote three noncollinear points in $\mathbb{R}^{2}$. Let $c$ denote a point on the line segment $A B, a$ a point on the line segment $B C$, and $b$ a point on the line segment $C A$, such that $\{a, b, c\} \cap\{A, B, C\}=\varnothing$; see panel (i) of Fig. 15. Let $f_{1}: \mathbb{R}^{2} \rightarrow \mathbb{R}^{2}$ denote the unique affine transformation such that

$$
f_{1}(A B C)=A b c
$$

by which we mean that $f_{1}$ maps $A$ to $A, B$ to $b$, and $C$ to $c$. Using the same notation, let affine transformations $f_{2}, f_{3}$, and $f_{4}$ be the ones uniquely defined by

$$
\begin{aligned}
& f_{2}(A B C)=a B c, \\
& f_{3}(A B C)=a b C, \\
& f_{4}(A B C)=a b c .
\end{aligned}
$$

Panel (ii) of Fig. 15 shows the images of the points $A, B, C$ under the four functions of the IFS, illustrating the special way that the four functions fit together. The attractor of $\mathcal{F}$ is the filled triangle with vertices at $A, B$, and $C$, illustrated in (iii) in Fig. 15.

If $\theta=\theta_{1} \theta_{2} \theta_{3} \cdots \in\{1,2,3,4\}^{\infty}$ is full, then according to Theorem $10.4, T_{\mathcal{F}, \theta}$ is a tiling of $\mathbb{R}^{2}$ by triangles. One such affine tiling is illustrated in Fig. 16. A related projective tiling is also shown in that figure. Note that, if $e$ is a common edge of two triangles $\Delta_{1}, \Delta_{2}$ in $T_{\mathcal{F}, \theta}$, then $e$ is the image of the same edge of the original triangle $A B C$ from $\Delta_{1}$ and $\Delta_{2}$, and if $v$ is a common vertex in $T_{\mathcal{F}, \theta}$ of two triangles $\Delta_{2}, \Delta_{2}$ in $T_{\mathcal{F}, \theta}$, then $v$ is the image of the same vertex of the original triangle $A B C$ from $\Delta_{1}$ and $\Delta_{2}$.

\subsection{Tilings from a graph IFS}

The construction of tilings from an IFS can be generalized to the construction of tilings from a graph IFS. There is considerable current interest in graph IFS and tilings corresponding to Rauzy fractals $[68,105,106]$. Hausdorff dimension of attractors associated 

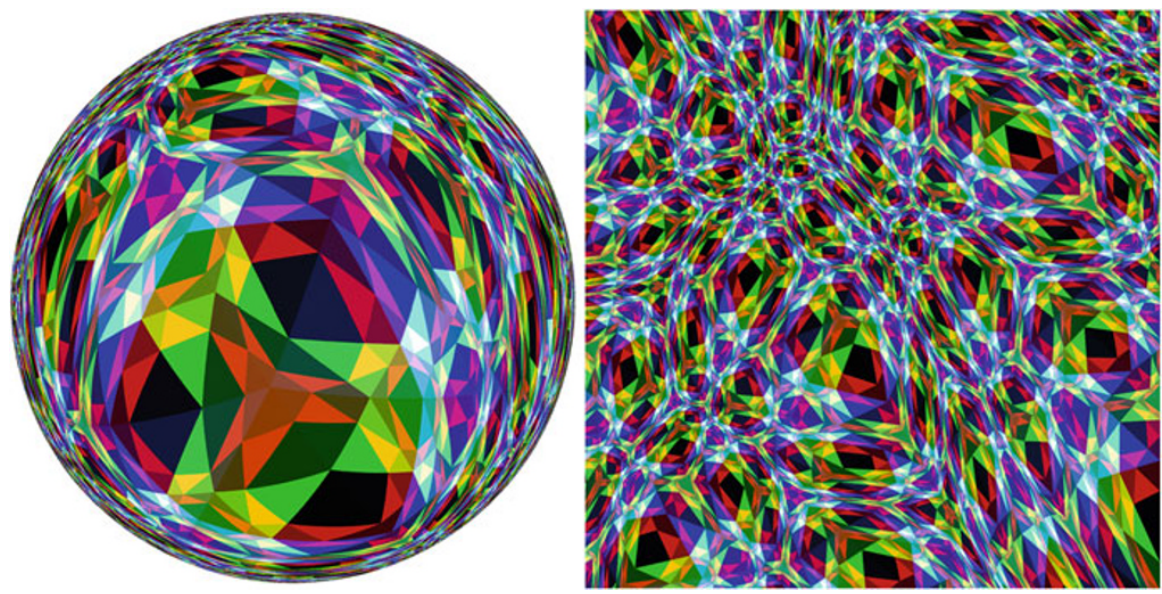

Fig. 16 The image on the right illustrates part of an affine tiling of the plane. All the tiles are triangular. The dark rectangles are unions of tiles of the same colour. The image on the left illustrates a related projective tiling, represented using the disk model for $\mathbb{R} P^{2}$ (color figure online)

with graph IFSs has been considered in [83]. Graph IFSs arise in connection with substitution tilings and number theory; see for example [2,43].

Let $H^{M}$ denote the $N$-fold Cartesian product of $M$ copies of $H(X)$. A graph IFS is a directed graph $\mathbb{G}$, possibly with loops and multiple edges, in which the vertices of $\mathbb{G}$ are labeled by $\{1,2, \ldots, M\}$ and each directed edge $e$ is labeled with a contraction $f_{e}: X \rightarrow X$. It is also assumed that $\mathbb{G}$ is strongly connected, i.e., that there is a directed path from any vertex to any other. Let $E_{i j}$ denote the set of edges from vertex $i$ to vertex $j$. Define the function

$$
F: H^{M} \rightarrow H^{M}
$$

as follows. If $\mathbf{Y}=\left(Y_{1}, Y_{2}, \ldots, Y_{M}\right) \in H^{M}$, then

$$
F(\mathbf{Y})=\left(F_{1}(\mathbf{Y}), F_{2}(\mathbf{Y}), \ldots, F_{M}(\mathbf{Y})\right)
$$

where

$$
F_{i}(\mathbf{Y})=\bigcup_{j=1}^{M} \bigcup_{e \in E_{i j}} f_{e}\left(Y_{j}\right)
$$

for $i=1,2, \ldots M$. It can be shown that $F$ is a contraction on $H^{M}$, and consequently has a unique fixed point or attractor $\mathbf{A}=\left(A_{1}, A_{2}, \ldots, A_{M}\right)$. An ordinary IFS is the special case of a graph IFS where $\mathbb{G}$ has exactly one vertex and all edges are loops.

Let $\mathbb{G}^{\prime}$ denote the graph obtained from $\mathbb{G}$ by reversing the directions on all of the edges. Assume that the graph IFS is non-overlapping. The concept of overlapping is defined exactly as for an IFS. For any directed (infinite) path $\theta=e_{1} e_{2} \cdots$ in $\mathbb{G}^{\prime}$, a 

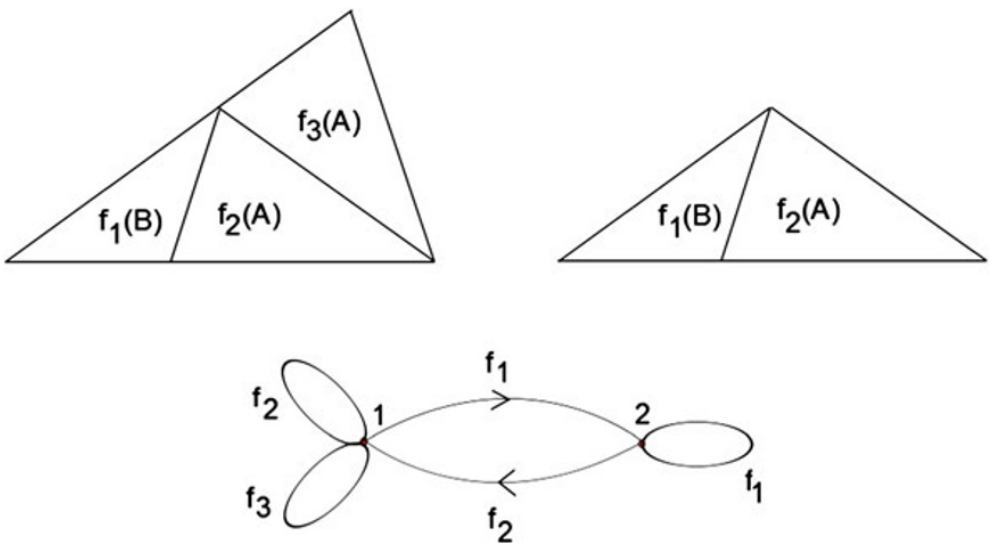

Fig. 17 Graph IFS for the Penrose tiles (color figure online)

tiling is constructed as follows. First extend previous notation so that $\theta_{k}=e_{k}, \theta \mid k=$ $e_{1} e_{2} \cdots e_{k}$ and $f_{\theta \mid k}=f_{e_{1}} \circ f_{e_{2}} \circ \cdots \circ f_{e_{k}},\left(f_{\theta \mid k}\right)^{-1}:=f_{e_{1}}^{-1} \circ f_{e_{2}}^{-1} \circ \cdots \circ f_{e_{k}}^{-1}$. Given any directed path $\omega$ of length $k$ in $\mathbb{G}$ that starts at the vertex at which $\theta \mid k$ terminates, let

$$
\begin{aligned}
t_{\theta, \omega} & =\left(\left(f^{-1}\right)_{\theta \mid k} \circ f_{\omega}\right)\left(A_{j}\right), \\
T_{\theta, k} & =\left\{t_{\theta, \omega}: \omega \in W_{k}\right\},
\end{aligned}
$$

where $j$ is the terminal vertex of the path $\omega$, and $W_{k}$ is the set of directed paths of length $k$ in $\mathbb{G}$ that start at the vertex at which $\theta \mid k$ terminates. Since, for any $\omega \in P_{k}$, we have

$$
\left(f^{-1}\right)_{\theta \mid k} \circ f_{\omega}=\left(f^{-1}\right)_{\theta \mid k} \circ\left(f_{\theta_{k+1}}\right)^{-1} \circ f_{\theta_{k+1}} \circ f_{\omega}=\left(f^{-1}\right)_{\theta \mid k+1} \circ f_{\theta_{k+1} \omega},
$$

the inclusion

$$
T_{\theta, k} \subset T_{\theta, k+1}
$$

holds for all $k$. Therefore

$$
T_{\theta}=T_{\mathbb{G}, \theta}:=\bigcup_{k=1}^{\infty} T_{\theta, k}
$$

is a tiling.

Example 10.10 (Penrose tilings of $\mathbb{R}^{2}$ ) In this example the graph $\mathbb{G}$ is given in Fig. 17 where, using the complex representation of $\mathbb{R}^{2}$, the functions are

$$
f_{1}(z)=\left(\frac{z}{\tau}+1\right) \omega_{1}, \quad f_{2}(z)=\frac{-z}{\tau}+\tau^{2}, \quad f_{3}(z)=\frac{z}{\tau} \omega_{3}+\tau^{2},
$$


where $\tau=(1+\sqrt{5}) / 2$ is the golden ratio and $\omega_{k}=\cos (k \pi / 5)+i \sin (k \pi / 5), 0 \leq$ $k \leq 9$, are the tenth roots of unity. The acute isosceles triangle $A$ and obtuse isosceles triangle $B$ in the figure have long and short sides in the ratio $\tau: 1$, and the angles are $\pi / 5,2 \pi / 5,2 \pi / 5$ and $\pi / 5, \pi / 5,3 \pi / 5$, respectively. The attractor of the graph IFS is the pair $(A, B)$ :

$$
\begin{aligned}
& A=f_{1}(B) \cup f_{2}(A) \cup f_{3}(A), \\
& B=f_{1}(B) \cup f_{2}(A) .
\end{aligned}
$$

Clearly, there are periodic tilings of the plane using copies of these tiles. However, if $\theta$ is a directed path in $\mathbb{G}$ and $T_{\mathbb{G}, \theta}$ tiles $\mathbb{R}^{2}$, then this is a non-periodic tiling. Although a Penrose tiling is usually given in terms of kites and darts or thin and fat rhombs, these are equivalent to tiling by the acute and obtuse triangles described in this example.

\section{Fractal continuation}

Fractal functions have a long history; see [121] and [90, Chapter 5]. They were introduced, in the form considered here, in [13]. In this section we discuss functions $f:[0,1] \rightarrow \mathbb{R}$ whose graph $G(f)$ is the attractor of an interpolation IFS, defined below. This includes well-known types of non-differentiable functions, including Takagi curves, Kiesswetter curves, Koch curves, and the Weierstrass nowhere differentiable function $f:[0,1] \rightarrow \mathbb{R}$, defined for $|\xi|<1$ by

$$
f(x)=\sum_{k=0}^{\infty} \xi^{k} \sin 2^{k+1} \pi x .
$$

Fractal functions are the basis of a constructive approximation theory for nondifferentiable functions. They have been developed both in theory and applications by many authors; see for example $[25,40,89,90,95,101,108,109,120]$. They provide an alternative view on wavelets, [40,53]. In [62] Hardin writes: “...compactly supported refinable functions (and thus compactly supported wavelets) are piecewise fractal interpolation functions. This perspective, although not expressed in this language, underlies much of the research on determining properties (such as regularity, approximation order, and linear independence) of a refinable function from its mask (cf. [50,84])."

In this section we précis a natural generalization of analytic continuation. Generalizing $[13,19,25]$, we first define (analytic) fractal function. The following theorem is an amalgam of some results proved in [37].

Theorem 11.1 Let $0=x_{0}<x_{1}<\cdots<x_{N}=1$ be real numbers with $N>1$, and let $\left\{y_{n}\right\}_{n=0}^{N} \subset \mathbb{R}$ so that $\mathcal{D}:=\left\{\left(x_{n}, y_{n}\right)\right\}_{n=0}^{N}$ is a set of data points. Let $X \subset \mathbb{R}^{2}$ be a pathwise connected neighbourhood of $\mathcal{D}$ and $\mathcal{W}$ be an injective IFS of the form

$$
\mathcal{W}=\left\{X ; w_{n}(x, y)=\left(\left(x_{n}-x_{n-1}\right) x+x_{n-1}, F_{n}(x, y)\right), n \in[N]\right\},
$$


where

(i) $F_{0}\left(x_{0}, y_{0}\right)=y_{0}, F_{n+1}\left(x_{0}, y_{0}\right)=F_{n}\left(x_{N}, y_{N}\right)=y_{n}$ for $n=1,2, \ldots, N-1$, and $F_{N}\left(x_{N}, y_{N}\right)=y_{N}$, and

(ii) there is an $s \in[0,1)$ and an $\mathcal{M} \in[0, \infty)$ such that

$$
\left|F_{n}(x, y)-F_{n}\left(x^{\prime}, y^{\prime}\right)\right| \leq \mathcal{M}\left|x-x^{\prime}\right|+s\left|y-y^{\prime}\right|
$$

for all $(x, y),\left(x^{\prime}, y^{\prime}\right) \in X, n \in[N]$.

Then the IFS $\mathcal{W}$ is contractive with (unique) attractor $A=G(f)$, the graph of a function $f:[0,1] \rightarrow \mathbb{R}$ such that $f\left(x_{n}\right)=y_{n}$ for all $n \in[N]$.

Definition 11.2 We call an IFS $\mathcal{W}$ of the form in Theorem 11.1 an interpolation IFS, and we call the function $f:[0,1] \rightarrow \mathbb{R}$ whose graph is the attractor of $\mathcal{W}$ a fractal function. If also $F_{n}(x, y)$ is real analytic for $(x, y) \in \mathcal{D}$, for $n=1,2, \ldots, N$, then we call $\mathcal{W}$ an analytic interpolation IFS, and we call $f$ an analytic fractal function.

Our nomenclature is reasonable because of the following theorem, which is proved in [37].

Theorem 11.3 If $f:[0,1] \rightarrow \mathbb{R}$ is analytic, then the graph $G(f)$ of $f$ is the attractor of an analytic interpolation IFS $\mathcal{W}=\left\{X ; w_{1}(x, y), w_{2}(x, y)\right\}$, where $X$ is a neighbourhood of $G(f)$.

The main new idea is to use a fractal blow-up technique to "continue" $f$. This technique, when applied to a real analytic function $f$, results in the analytic continuation of $f$ in the usual sense. Let $\theta \in \Omega$ and $G=G(f)$. Define

$$
G_{\theta \mid k}:=\left\{(x, y) \in X: w_{\theta_{k}} \circ w_{\theta_{k-1}} \circ \circ w_{\theta_{1}}(x, y) \in G\right\} .
$$

Theorem 11.4 With notation as above,

$$
G \subset G_{\theta \mid 1} \subset G_{\theta \mid 2} \subset \cdots
$$

Moreover, $G_{\theta \mid k}$ is the graph of a continuous function $f_{\theta \mid k}$ whose domain is

$$
I_{\theta \mid k}:=\left(L^{-1}\right)_{\theta \mid k}([0,1])=L_{\theta_{1}}^{-1} \circ L_{\theta_{2}}^{-1} \circ \cdots \circ L_{\theta_{k}}^{-1}([0,1]) \cap P(X),
$$

where $L_{n}(x)=\left(x_{n}-x_{n-1}\right) x+x_{n-1}$ for all $n \in[N]$, and $P(X)$ is the projection of $X$ on the $x$-axis.

Definition 11.5 Referring to Theorem 11.4, for $\theta \in \Omega$ let $I_{\theta}=\cup_{k} I_{\theta \mid k}$ and $f_{\theta}: I_{\theta} \rightarrow$ $\mathbb{R}$ be defined by $\left.f_{\theta}\right|_{I_{\theta \mid k}}=f_{\theta \mid k}$. Then $f_{\theta}: I_{\theta} \rightarrow \mathbb{R}$ is called the fractal continuation of $f$ with respect to $\theta$ (and $\mathcal{W}$ ), and $\left\{f_{\theta}: \theta \in \mathcal{I}^{\infty}\right\}$ is the set of fractal continuations (w.r.t. $\mathcal{W}$ ) of the fractal function $f$. If the interpolation IFS $\mathcal{W}$ is analytic, then the phrase analytic fractal continuation replaces the phrase "fractal continuation" in the previous sentence. 
Theorem 11.6 below states some facts about fractal continuations. According to statement (2), analytic fractal continuation of an analytic function is unique, not depending on the string $\theta \in \Omega$, and is the standard analytic continuation. Statement (3) is of practical value, as it implies that stable methods, such as the chaos game algorithm, for computing numerical approximations to attractors, may be used to compute fractal continuations. A fractal function may possess uncountably many distinct continuations.

Theorem 11.6 With the notation as above, for all $\theta \in \Omega$ the following statements hold:

(1) $f_{\theta}(x)=f(x)$ for all $x \in[0,1]$.

(2) If both $\mathcal{W}$ and $f$ are analytic on $[0,1]$, then $f_{\theta}(x)=\widetilde{f}(x)$ for all $x \in I_{\theta}$, where $\widetilde{f}$ is the (unique) real analytic continuation of $f$.

(3) If $X=\mathbb{R}^{2}$ and $\mathcal{W}$ is invertible, then for all $k \in \mathbb{N}$ the IFS

$$
\mathcal{W}_{\theta \mid k}:=\left\{\mathbb{R}^{2} ; w_{\theta \mid k}^{-1} \circ w_{n} \circ\left(\left(w^{-1}\right)_{\theta \mid k}\right)^{-1}\right\}=\left\{\mathbb{R}^{2} ; w_{\theta \mid k}^{-1} \circ w_{n} \circ w_{\hat{\theta \mid k}}\right\}
$$

has attractor $G_{\theta \mid k}=G\left(f_{\theta \mid k}\right)$.

In [37] we conjecture that the set of continuations of an analytic fractal function is independent of the analytic interpolation IFS. This conjecture requires more precision than we give here; roughly, it states that if two analytic interpolation IFSs $\mathcal{W}$ and $\widetilde{\mathcal{W}}$, both with the same space $X$, have the same attractor $G(f)$, and if $f_{\theta}$ is a continuation of $f$ w.r.t. $\mathcal{W}$, then there is a continuation $\widetilde{f_{\tilde{\theta}}}$ of $f$ w.r.t. $\widetilde{\mathcal{W}}$, such that $\widetilde{f_{\tilde{\theta}}}=f_{\theta}$. In [37] this conjecture is proved for some cases where it is further assumed that $G(f)$ is rectifiable.

Example 11.7 Let $G^{(p)}$ be the attractor of the affine IFS

$\mathcal{W}_{p}=\left\{\mathbb{R}^{2}, w_{1}(x, y)=(0.5 x, 0.5 x+p y), w_{2}(x, y)=(0.5 x+1,-0.5 x+p y+1)\right\}$,

where $p \in(-1,0) \cup(0,1)$ is a parameter.

The attractor $G^{(p=0.25)}$ is the graph of the analytic function $f:[0,2] \rightarrow \mathbb{R}$, where

$$
f(x)=x(2-x)
$$

and, according to statement (2) of Theorem 11.6 the unique continuation is $f_{\theta}(x)=$ $x(2-x)$ with domain $[0, \infty)$ if $\theta=\overline{1}$, domain $(-\infty, 2]$ if $\theta=\overline{2}$, and domain $(-\infty, \infty)$ otherwise.

When $p=0.3$ the attractor is the graph of a non-differentiable function, and there are non-denumerably many distinct continuations $f_{\theta}:(-\infty, \infty) \rightarrow \mathbb{R}$. Figure 18 shows some of these continuations, restricted to the domain $[-20,20]$. More precisely, Fig. 18 shows the graphs of $f_{\theta \mid 4}(x)$ for all $\theta \in[2]^{\infty}$. The continuation $f_{\overline{1}}(x)$, on the right in black, coincides exactly, for $x \in[2,4]$, with all continuations of the form $f_{1 \rho}(x)$ with $\rho \in[2]^{\infty}$. To the right of center: the blue curve is $G_{2111}$, the green curve is $G_{2211}$, and the red curve is $G_{2221}$. On the left: the lowest curve (part red, part blue) 


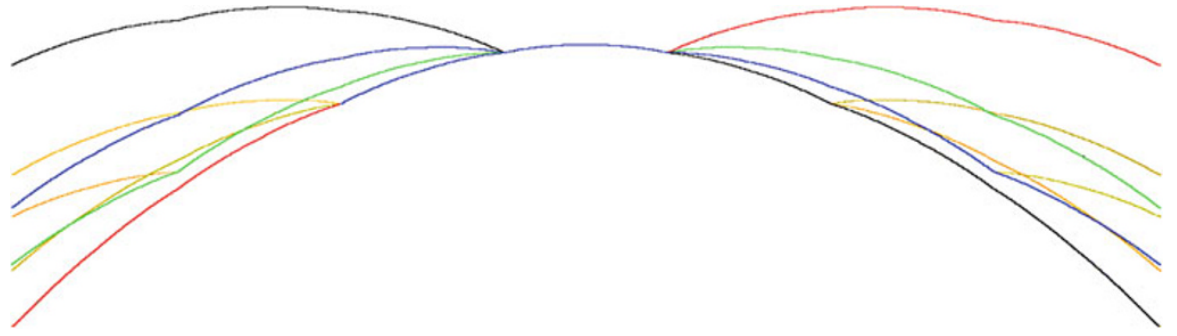

Fig. 18 See Example 11.7 (color figure online)

Fig. 19 Added detail for part of

Fig. 18 showing additional continuations near the ends of the original function (color figure online)
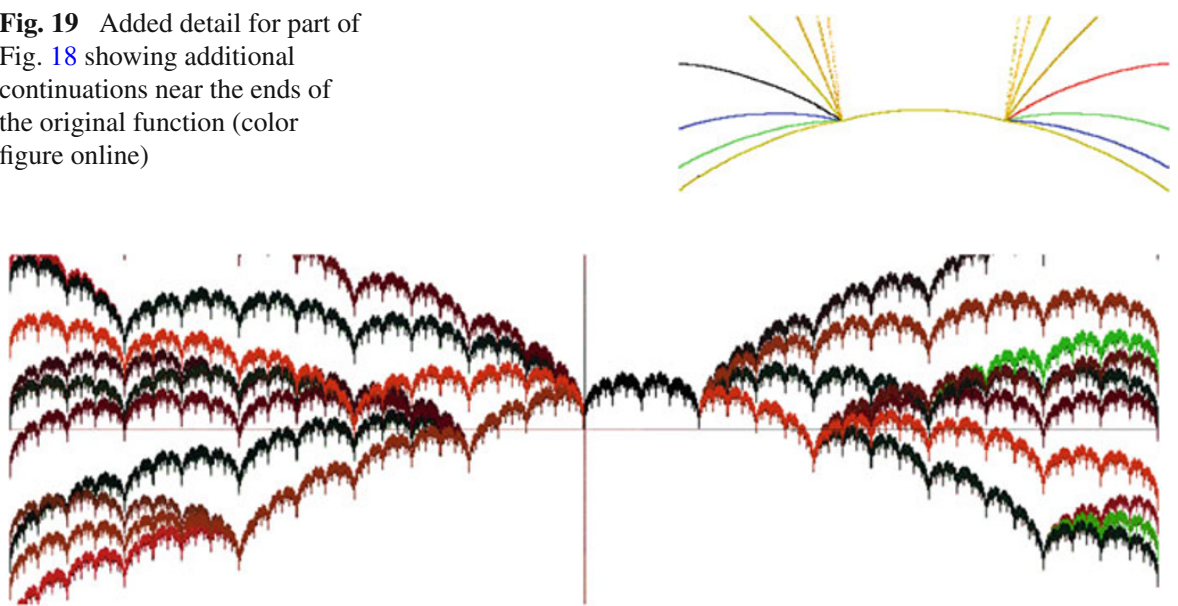

Fig. 20 See Example 11.7. The colors help to distinguish the different continuations of the fractal function whose graph is illustrated in black near the center of the image (color figure online)

is $G_{2222}$, the green curve is $G_{1222}$, the blue curve is $G_{1122}$, and the black curve is $G_{112}$. Also see Fig. 19 .

The attractor $G^{(p=0.8)}$ is the graph of a fractal function $f^{(p=0.8)}$ whose graph has Minkowski dimension $(2-\ln (5 / 4) / \ln 2)$. This $\operatorname{graph} G^{(p=0.8)}$ is illustrated in the middle of Fig. 20. The window for Fig. 20 is $[-10,10] \times[-10,10] \subset \mathbb{R}^{2}$, and $f^{(p=0.8)}$ is the (unique) black object whose domain is [0,2]. Figure 20 shows all continuations $f_{\theta \mid 4}^{(p=0.8)}(x)$ for $\theta \in[2]^{\infty}$.

\section{Conley attractors}

An IFS $\mathcal{F}=\left\{X ; f_{1}\right\}$ defines a discrete dynamical system $f_{1}: X \rightarrow X$. There are various definitions of an attractor of a dynamical system. Some definitions use measure theory to describe the probable asymptotic behaviour of orbits, see [85], while others are purely topological. Among the latter is the Conley attractor, which is interesting because of its generality, the "landscape picture" [48] for both discrete and continuous flows, and its role in the fundamental theorem of dynamical systems [98]. In this 
section we define the Conley attractor of an IFS, discuss the existence of attractorrepeller pairs (Theorem 12.5) and their relation to the dynamics of the IFS (Theorem 12.7), and explain Conley's "landscape picture" as it applies to an IFS (Theorem 12.9). Our presentation is a simplified version of the elegant work of McGehee and Wiandt $[92,126]$ about iterated closed relations on compact Hausdorff spaces.

The notion of a Conley attractor generalizes Definition 2.2, which is much used in the fractal geometry literature because it derives from the situation for a contractive IFS. The following three definitions look almost identical to Definitions 2.2, 2.3, and 2.4, the crucial differences being that a Conley attractor $A$ may be empty and orbits of sets under $F: 2^{X} \rightarrow 2^{X}$ are "absorbed" by $A$ rather than converging, in a cascade, to $A$.

Definition 12.1 A Conley attractor of the IFS $\mathcal{F}$ is a compact set $A \subset X$ such that

(1) $F(A)=A$ and

(2) there is an open set $U \subset X$ such that $A \subset U$ and $\lim _{k \rightarrow \infty} F^{k}(\bar{U})=A$ where the limit is with respect to the Hausdorff metric on $H$.

In the terminology of [71, Definition 4.4.1], an attractor of an IFS $\mathcal{F}$ is a nonempty asymptotically stable Conley attractor of $\mathcal{F}$.

Definition 12.2 The basin $B$ of a Conley attractor $A$ of the IFS $\mathcal{F}$ is the largest open set $U$ such that (2) in Definition 12.1 is true.

Definition 12.3 The dual repeller of a Conley attractor $A$ of the IFS $\mathcal{F}$ is the set $A^{*}:=X \backslash B$ where $B$ is the basin of $A$.

Theorem 12.4 gives equivalent characterizations of a Conley attractor.

Theorem 12.4 For an IFS $\mathcal{F}$ on a compact metric space $X$, a compact set $A$ is a Conley attractor of $\mathcal{F}$ if and only if either of the following two equivalent conditions hold:

1. there is an open set $U \subset X$, with $A \subset U$, such that $A=\bigcap_{K \geq 1} \overline{\bigcup_{k \geq K} F^{k}(U)}$;

2. there is an open set $U \subset X$, with $A \subset U$, such that $F(\bar{U}) \subset U^{o}$ and $A=$ $\bigcap_{k=0}^{\infty} F^{k}(\bar{U})$.

A simple proof of Theorem 12.4 can be found in [36], but greater generality and other characterizations of Conley attractors and related entities appear in [81,91,92].

The empty set is always a Conley attractor of an IFS, and $X$ is an attractor if $\mathcal{F}$ contains at least one surjective function. There exist IFSs, even consisting of a single function on the circle, that have infinitely many Conley attractors [36]. An attractor in the sense of Definition 2.2 is a Conley attractor, but a nonempty Conley attractor is not necessarily an attractor in the sense of Definition 2.2, even for the case of an IFS consisting of a single function on the circle.

The following theorem expresses a symmetrical relationship between Conley attractors and dual repellers.

Theorem 12.5 Let $\mathcal{F}$ be an invertible IFS on a compact metric space X. If $A$ is a Conley attractor of $\mathcal{F}$ with basin $B$, then $A^{*}:=X \backslash B$ is a Conley attractor of $\mathcal{F}^{-1}$ with basin $X \backslash A$. 
If $\mathcal{F}$ is contractive with attractor $A$, then $\mathcal{F}$ possesses a unique dual repeller $A^{*}=\emptyset$. Recall that some examples of attractor-repeller pairs are exhibited in Sect. 2. A projective IFS that possesses an attractor that avoids a hyperplane always has a nonempty dual repeller.

The notion of a chain for an IFS is based on the notion of a chain for a single function [48].

Definition 12.6 Let $\varepsilon>0$ and let $\mathcal{F}$ be an IFS on $X$. An $\varepsilon$-chain for $\mathcal{F}$ is a sequence of points $\left\{x_{i}\right\}_{i=0}^{n}, n>0$, in $X$ such that for each $i \in\{0,1,2, \ldots, n-1\}$ there is an $f \in \mathcal{F}$ such that $d\left(x_{i+1}, f\left(x_{i}\right)\right)<\varepsilon$. A point $x \in X$ is chain-recurrent for the IFS $\mathcal{F}$ if, for every $\varepsilon>0$, there is an $\varepsilon$-chain $\left\{x_{i}\right\}_{i=0}^{n}$ for $\mathcal{F}$ such that $x_{0}=x_{n}=x$. The set of all chain recurrent points for $\mathcal{F}$ is denoted by $\mathcal{R}:=\mathcal{R}_{\mathcal{F}}$.

We refer to the following as the Conley-McGehee-Wiandt (CMW) theorem due to their analogs of Theorem 12.7 in the context of dynamical systems and iterated closed relations. The present version could also be deduced from the work of Akin [1]; a direct proof is given in [36].

Theorem 12.7 [CMW] Let $\mathcal{F}$ be an invertible IFS on a compact metric space X. If $\mathcal{U}$ denotes the set of Conley attractors of $\mathcal{F}$ and $\mathcal{R}_{\mathcal{F}}$ denotes the set of chain recurrent points of $\mathcal{F}$, then

$$
\mathcal{R}_{\mathcal{F}}=\bigcap_{A \in \mathcal{U}}\left(A \cup A^{*}\right) .
$$

Finally Conley's "landscape picture" is described, as it applies to an invertible IFS $\mathcal{F}$ on a compact metric space. Suppose that $A$ is an attractor of $\mathcal{F}$.

Definition 12.8 A continuous function $\mathcal{L}: X \rightarrow[0,1]$ is a Liapunov function for $\mathcal{F}$ if $\mathcal{L}(f(x)) \leq \mathcal{L}(x)$ for all $f \in \mathcal{F}$, for all $x \in X$. It is called a strict Liapunov function for $\mathcal{F}$ if it is a Liapunov function for $\mathcal{F}$ and $\mathcal{L}(f(x))<\mathcal{L}(x)$ whenever $x \notin \mathcal{R}_{\mathcal{F}}$.

A chain component of an invertible IFS $\mathcal{F}$ is an equivalence class of the following equivalence relation on $\mathcal{R}_{\mathcal{F}}$. Points $x$ and $y$ are equivalent if and only if, for each $\varepsilon>0$, there is an $\varepsilon$-chain for $\mathcal{F}$ and an $\varepsilon$-chain for $\mathcal{F}^{-1}$, each of which contains both $x$ and $y$. See [92, Definition 9.26] for the definition of chain component when $\mathcal{F}$ is not invertible.

Theorem 12.9 If $\mathcal{F}$ is an IFS on a compact metric space $X$, then there exists a strict Liapunov function $\mathcal{L}$ for $\mathcal{F}$. Moreover $\mathcal{L}\left(\mathcal{R}_{\mathcal{F}}\right)$ is a nowhere dense subset of $[0,1]$, and $\mathcal{L}$ is constant on the chain components.

We have simplified the original statement of Theorem 12.9 in [126, Theorem 6.19] by noting that a compact metric space is second countable.

Here is the "landscape picture" that we obtain. Given an IFS $\mathcal{F}$ with an attractor $A$, there is a "landscape" or surface or real valued height function over $X$ with all the points of $A$ lying at "sea level" at height 0 . All other points are higher. If one 
follows any chaos game orbit, starting anywhere on $X$, one will either travel "downhill" or stay at the same height. If one stays at the same positive height above sea-level from one step to the next, then one is dancing around on a chain component that is not the original attractor. If one follows an orbit that starts on the complement of the chain recurrent set, then one will travel downhill until one reaches a chain component.

By way of an example, consider the Julia set $J_{\lambda}$ for the quadratic map $z \longmapsto z^{2}-\lambda$ on the Riemann sphere $\widehat{\mathbb{C}}=\mathbb{R}^{2} \cup\{\infty\}$. For $\lambda \in(-0.25,0.75)$, it is well-known that $J_{\lambda}$ is a Jordan curve that divides $\widehat{\mathbb{C}}$ into two compact components, $C_{0}:=$ the component of $\widehat{\mathbb{C}} \backslash J_{\lambda}$ that contains the origin, and $C_{\infty}:=$ the component of $\widehat{\mathbb{C}} \backslash J_{\lambda}$ that contains $\infty$. Moreover, $J_{\lambda}$ is an attractor of the IFS $\mathcal{F}_{\lambda}=\left\{\mathbb{\mathbb { C }} ; f_{1}(z)=\sqrt{z+\lambda}, f_{2}(z)=\right.$ $-\sqrt{z+\lambda}\}$, where an appropriate definition of a branch cut needs to be made, see [19]. This IFS possesses the following Conley attractors
(i) $A_{1}=\widehat{\mathbb{C}}$;
(ii) $A_{2}=\overline{C_{0}}$;
(iii) $A_{3}=\overline{C_{\infty}}$;
(iv) $A_{3}=J_{\lambda}$.

The corresponding dual repellers are
(i) $A_{1}^{*}=\emptyset$;
(ii) $A_{2}^{*}=\{\infty\}$;
(iii) $A_{3}^{*}=\left\{Z_{0}\right\}$;
(iv) $A_{3}^{*}=\left\{Z_{0}\right\} \cup\{\infty\}$,

where $Z_{0}=0.5+\sqrt{1+4 \lambda} / 2$. The chain recurrent set is $\mathcal{R}_{\mathcal{F}_{\lambda}}=\left\{Z_{0}\right\} \cup J_{\lambda} \cup \infty$. In this example, a Liapunov function $\mathcal{L}: \widehat{\mathbb{C}} \rightarrow[0,1]$, as guaranteed by Theorem 12.9, is continuous and such that $\mathcal{L}\left(J_{\lambda}\right)=0, \mathcal{L}\left(Z_{0}\right)=\mathcal{L}(\infty)=1$. What is the behaviour of any chaos game orbit $\left\{z_{n}\right\}_{n=0}^{\infty}$ ? For $z_{0} \notin\left\{Z_{0}, \infty\right\} \cup J_{\lambda}$, the set of values $\left\{\mathcal{L}\left(x_{n}\right)\right\}_{n=0}^{\infty}$ is strictly decreasing, and converges to 0 as $n \rightarrow \infty$.

\section{Concluding remarks}

This survey is focused on an investigation of the structure of IFSs, in the case where the functions are non-conformal, as well as the more familiar conformal case. The concepts in Sections 3 through 6 and Section 8 are basic to IFS theory-the role of contractivity on the existence of an attractor, the representation and emergence of the attractor, the addressing of points of the attractor, and the connectedness of the attractor. Although the concepts are foundational, the results in those sections are very recent and, in some cases, unexpected. The notion of the kneading invariant of an IFS attractor, introduced in Section 7, is important because it links IFS theory to dynamical systems. The ideas in Sections 9 through 12-fractal transformations, tilings, continuation of fractal functions, and properties of attractor-repeller pairs of a general IFSs - are perhaps the most promising for future research, both for theory and for applications.

Acknowledgment We thank a referee for helpful suggestions.

Open Access This article is distributed under the terms of the Creative Commons Attribution License which permits any use, distribution, and reproduction in any medium, provided the original author(s) and the source are credited. 


\section{References}

1. Akin, E.: The General Topology of Dynamical Systems. American Mathematical Society, Providence (1993)

2. Akiyama, S., Thuswaldner, J.M.: A survey on topological properties of tiles related to number systems. Geom. Dedic. 109, 89-105 (2004)

3. Akiyama, S.: Symbolic dynamical system and number theoretical tilings. Selected papers on analysis and related topics. Am. Math. Soc. Transl. Ser. 223, 97-113 (2008)

4. Atkins, R., Barnsley, M.F., Wilson, D.C., Vince, A.: A characterization of point-fibred affine iterated function systems. Topol. Proc. 38, 189-211 (2010)

5. Bandt, C.: Self-similar sets 5. Integer matrices and fractal tilings of $\mathbb{R}^{n}$. Proc. Am. Math. Soc. 112, 549-562 (1991)

6. Bandt, C.: On the Mandelbrot set for pairs of linear maps. Nonlinearity 15, 1127-1147 (2002)

7. Bandt, C.: Bernoulli convolutions and branching dynamical systems. Abstracts for International Conference on Advances in Fractals and Related Topics, Hong Kong, December (2012)

8. Bandt, C., Gummelt, P.: Fractal Penrose tilings I. Construction and matching rules. Aequ. Math. 53, 295-307 (1997)

9. Bandt, C., Hung, N.V.: Fractal n-gons and their Mandelbrot sets. Nonlinearity 21, 2653-2670 (2008)

10. Bandt, C., Keller, K.: Self-similar sets 2. A simple approach to the topological structure of fractals. Math. Nachr. 154, 27-39 (1991)

11. Bandt, C., Rao, H.: Topology and separation of self-similar fractals in the plane, Preprint (2012)

12. Barlow, M.T., Bass, R.F.: Brownian motion and harmonic analysis on Sierpinski carpets. Can. J. Math. 54, 673-744 (1999)

13. Barnsley, M.F.: Fractal functions and interpolation. Constr. Approx. 2, 303-329 (1986)

14. Barnsley, M.F.: Fractal image compression. Not. Am. Math. Soc. 43, 657-662 (1996)

15. Barnsley, M.F.: Theory and applications of fractal tops. In: Levy-Vehel, J., Lutton, E. (eds.) Fractals in Engineering: New Trends in Theory and Applications. Springer, London (2005)

16. Barnsley, M.F.: SuperFractals. Cambridge University Press, Cambridge (2006)

17. Barnsley, M.F.: Transformations between self-referential sets. Am. Math. Month. 116, 291-304 (2009)

18. Barnsley, M.F.: The life and survival of mathematical ideas. Not. AMS 57, 10-22 (2010)

19. Barnsley, M.F.: Fractals Everywhere. Academic Press, Dublin (1988). (2nd Edition, Morgan Kaufmann 1993; 3rd Edition, Dover Publications, 2012)

20. Barnsley, M.F., Frame, M. (eds.): The influence of Benoît B. Mandelbrot on Mathematics. Not. Am. Math. Soc. 59(9), 1208-1221 (2012)

21. Barnsley, M.F., Harding, B., Igudesman, K.: How to transform and filter images using iterated function systems. SIAM J. Imaging Sci. 4(4), 1001-1028 (2011)

22. Barnsley, M.F., Harding, B., Vince, A.: The entropy of an overlapping dynamical system. Ergod. Theory Dyn. Syst. (2013, to appear)

23. Barnsley, M.F., Harding, B.: Three-dimensional fractal homeomorphisms. In: Memorial book in honour of Benoit Mandelbrot. World Scientific, Singapore (2012, to appear)

24. Barnsley, M.F., Harrington, A.N.: A Mandelbrot set for pairs of linear maps. Phys. D 15, 421-32 (1985)

25. Barnsley, M.F., Harrington, A.N.: The calculus of fractal interpolation functions. J. Approx. Theory 57, 14-34 (1989)

26. Barnsley, M.F., Hutchinson, J.E., Stenflo, Ö.: A fractal valued random iteration algorithm and fractal hierarchy. Fractals 13(2), 111-146 (2005)

27. Barnsley, M.F., Hutchinson, J.E., Stenflo, Ö.: V-variable fractals: fractals with partial similarity. Adv. Math. 218, 2051-2088 (2008)

28. Barnsley, M.F., Hutchinson, J.E., Stenflo, Ö.: V-variable fractals: dimension results. Forum Math. 24, 445-470 (2012)

29. Barnsley, M.F., Lesniak, K.: The chaos game on a general iterated function system from a topological point of view, arXiv:1203.0481 (2012)

30. Barnsley, M.F., Leśniak, K.: On the continuity of the Hutchinson operator, arXiv:1202.2485 (2012)

31. Barnsley, M.F., Wilson, D.C., Lesniak, K.: Some recent progress concerning topology of fractals. In: Arhangelskii, A.V., van Mill, J. (eds.) Recent Progress in Topology III (2013, to appear) 
32. Barnsley, M.F., Vince, A.: The eigenvalue problem for linear and affine iterated function systems. Linear Algebra Appl. 435, 3124-3138 (2011)

33. Barnsley, M.F., Vince, A.: The chaos game on a general iterated function system. Ergod. Theory Dyn. Syst. 31, 1073-1079 (2011)

34. Barnsley, M.F., Vince, A.: Real projective iterated function systems. J. Geom. Anal. 22, 1137-1172 (2012)

35. Barnsley, M.F., Vince, A.: Fractal homeomorphism for bi-affine iterated function sytems. Int. J. Appl. Nonlinear Sci. 1, 3-19 (2012)

36. Barnsley, M.F., Vince, A.: The Conley attractors of an iterated function system. Bull. Aus. Math. Soc. (2013, to appear)

37. Barnsley, M.F., Vince, A.: Fractal continuation, Constructive Approximation (2013, to appear)

38. Barnsley, M.F., Vince, A.: Fractal tiling. In preparation (2013)

39. Beaucoup, F., Borwein, P., Boyd, D., Pinner, C.: Multiple roots of [-1,1] power series. J. Lond. Math. Soc. 57(2), 135-147 (1998)

40. Berger, M.A.: Random affine iterated function systems: curve generation and wavelets. SIAM Rev. 34, 361-385 (1992)

41. Berger, M.A., Wang, Y.: Bounded semigroups of matrices. Linear Algebra Appl. 66, 21-27 (1992)

42. Berry, M.V.: Benefiting from fractals (a tribute to Benoit Mandelbrot). Proc. Symp. Pure Math. 72, 31-33 (2004)

43. Berthé, V., Siegel, A., Steiner, W., Surer, P., Thuswaldner, J.M.: Fractal tiles associated with shift radix systems. Adv. Math. 226, 139-175 (2011)

44. Bessaga, C.: On the converse of the Banach fixed point principle. Colloq. Math. 7, 41-43 (1959)

45. Bousch, T.: Sur quelques problèmes de dynamique holomorphe, Ph.D. thesis, Orsay (1992). http:// topo.math.u-psud.fr/ bousch

46. Chousionis, V.: Directed porosity on conformal IFS and weak convergence of singular integrals. Ann. Acad. Sci. Fenn. Math. 34, 215-232 (2009)

47. Collet, P., Eckmann, J.P.: Iterated Maps of the Interval as Dynamical Systems. Birkhäuser, Basel (1980)

48. Conley, C.: Isolated invariant sets and the Morse index. CBMS Regional Conference Series, vol. 38. American Mathematical Society, New York (1978)

49. Daubechies, I., Lagarias, J.C.: Sets of matrices all infinite products of which converge. Linear Algebra Appl. 161, 227-263 (1992)

50. Daubechies, I., Lagarias, J.C.: Two-scale difference equations II. Local regularity, infinite products of matrices, and fractals. SIAM J. Math. Anal. 23, 1031-1092 (1992)

51. Deng, Q.R., Lau, K.S.: Open set condition and post-critically finite self-similar sets. Nonlinearity 21, 1227-1232 (2007)

52. Doeblin, W., Fortet, R.: Sur les chaines a liaisons complètes. Bull. Soc. Math. de France 65, 132-148 (1937)

53. Donovan, G., Geronimo, J.S., Hardin, D.P., Massopust, P.R.: Construction of orthogonal wavelets using fractal interpolation functions. SIAM J. Math. Anal. 27(4), 1158-1192 (1996)

54. Douady, A., Hubbard, J.: Iteration des polynomes quadratiques complexes. R. Acad. Sci. Paris 294, 123-126 (1982)

55. Elekes, M.: On a converse to Banach's fixed point theorem. Proc. Am. Math. Soc. 137, 3139-3246 (2009)

56. Falconer, K.: Fractal Geometry-Mathematical Foundations and Applications. Wiley, Chichester (1990)

57. FrangoCamera, an iPad application (2012), and Frango Free, an iPhone application (2013). http:// www.frangostudio.com

58. Gelbrich, G.: Crystallographic reptiles. Geom. Dedic. 51, 235-256 (1994)

59. Gröchenig, K., Madych, W.R.: Multiresolution analysis, Haar bases and self-similar tilings of $\mathbb{R}^{n}$. IEEE Trans. Inf. Theory 38, 556-568 (1992)

60. Gröchenig, K., Haas, A.: Self-similar lattice tilings. J. Fourier Anal. Appl. 1, 131-170 (1994)

61. Hambly, B.M.: Asymptotics for functions associated with heat flow on the Sierpinski carpet. Can. J. Math. 63, 153-180 (2011)

62. Hardin, D.P.: Wavelets are piecewise fractal interpolation functions. In: Barnsley, M.F., Saupe, D., Vrscay, E.R. (eds.) The IMA Volumes in Mathematics and its Applications: Fractals in Multimedia, vol. 132, pp. 121-135. Springer, New York (2002) 
63. Henrikson, J.: Completeness and total boundedness of the Hausdorff metric. MIT Undegraduate J. Math. 1, 69-79 (1999)

64. Hochman, M.: Progress on self similar sets and measures with overlaps. Abstracts for International Conference on Advances in Fractals and Related Topics, Hong Kong, December (2012)

65. Hutchinson, J.: Fractals and self-similarity. Indiana Univ. Math. J. 30, 713-747 (1981)

66. Fractile (a Julia set and Mandelbrot set calculator for the iPad and iPhone). http://www.fractile.net

67. Janos̆ L.: A converse of Banach's contraction theorem. Proc. Am. Math. Soc. 18, 287-289 (1967)

68. Jolivet, T.: Fundamental groups of Rauzy fractals. Abstracts for International Conference on Advances in Fractals and Related Topics, Hong Kong, December (2012)

69. Kameyama, A.: Distances on topological self-similar sets. Proc. Symp. Pure Math. 71(1), 117-129 (2004)

70. Kenyon, R.: The construction of self-similar tilings. Geom. Funct. Anal. 6, 471-488 (1996)

71. Kieninger, B.: Iterated Function Systems on Compact Hausdorff Spaces, Ph.D. Thesis, Augsburg University, Berichte aus der Mathematik, Shaker-Verlag, Aachen (2002)

72. Kigami, J.: Harmonic calculus on p.c.f. self-similar sets. Trans. Am. Math. Soc. 335, 721-755 (1993)

73. Kigami, J.: Analysis on Fractals. Cambridge University Press, Cambridge (2001)

74. Kigami, J.: Measurable Riemannian geometry on the Sierpinski gasket: the Kusuoka measure and the Gaussian heat kernel estimate. Math. Ann. 340(4), 781-804 (2008)

75. Kirat, I., Lau, K.-S., Rao, H.: Expanding polynomials and connectedness of self-affine tiles. Discr. Comput. Geom. 31, 275-286 (2004)

76. Kunze, H.E., La Torre, D., Vrscay, E.R.: Contractive multifunctions, fixed point inclusions and iterated multifunction systems. J. Math. Anal. Appl. 330, 159-173 (2007)

77. Kusuoka, S.: Dirichlet forms on fractals and products of random matrices. Publ. Res. Inst. Math. Sci. 25, 659-680 (1989)

78. Lagarias, J., Wang, Y.: Self-affine tiles in $\mathbb{R}^{n}$. Adv. Math. 121, 21-49 (1996)

79. Lasota, A., Myjak, J.: Attractors of multifunctions. Bull. Polish Acad. Sci. 48(3), 319-334 (2000)

80. Leader, S.: A topological characterization of Banach contractions. Pacific J. Math. 69, 461-466 (1977)

81. Leśniak, K.: Stability and invariance of multivalued iterated function systems. Math. Slovaca 53(4), 393-405 (2003)

82. Leung, K.S.: Connectedness of self-affine sets associated with 3-digit sets. Abstracts for International Conference on Advances in Fractals and Related Topics, Hong Kong, December (2012)

83. Mauldin, R.D., Urbański, M.: Graph Directed Markov Systems: Geometry and Dynamics of Limit Sets. Cambridge University Press, Cambridge (2005)

84. Micchelli, C.: Mathematical Aspects of Geometric Modelling, vol. 65. SIAM, Philadelphia (1994)

85. Milnor, J.: On the concept of attractor. Comm. Math. Phys. 99, 177-195 (1985)

86. Milnor, J., Thurston, W.: On iterated maps of the interval. Lect. Notes Math. 1342, 465-563 (1988)

87. Mandelbrot, B.: Form. Chance and Dimension. W. H. Freeman, San Francisco (1977)

88. Mandelbrot, B.: The Fractal Geometry of Nature. W. H. Freeman, San Francisco (1983)

89. Massopust, P.: Fractal Functions, Fractal Surfaces, and Wavelets. Academic Press, New York (1995)

90. Massopust, P.: Interpolation and Approximation with Splines and Fractals. Oxford University Press, Oxford (2010)

91. McGehee, R.P.: Attractors for closed relations on compact Hausdorff spaces. Indiana U. Math. J. 41, 1165-1209 (1992)

92. McGehee, R.P., Wiandt, T.: Conley decomposition for closed relations. Diff. Equ. Appl. 12, 1-47 (2006)

93. Mera, M.E., Moran, M., Preiss, D.: Zajíček, L.: Porosity, $\sigma$-porosity and measures. Nonlinearity 16, 247-255 (2003)

94. Nadler, S.: Multi-valued contraction mappings. Pacific J. Math. 30, 475-488 (1969)

95. Navascues, M.A.: Fractal polynomial interpolation. Zeitschrift für Analysis u. i. Anwend 24, 401-414 (2005)

96. Ni, T., Wen, Z.: Open set condition for graph-directed self-similar structure, Preprint (2012)

97. Nikiel, S.: Iterated Function Systems for Real Time Image Synthesis. Springer, London (2007)

98. Norton, D.E.: The fundamental theorem of dynamical systems. Comment. Math. Univ. Carolin. 36, 585-597 (1995)

99. Onicescu, O., Mihoc, G.: Sur les chaînes des variables statistiques. Bull. Sci. Math. de France 59, 174-192 (1935) 
100. Parry, W.: Symbolic dynamics and transformations of the unit interval. Trans. Am. Math. Soc. 122, 368-378 (1965)

101. Prasad, S.A.: Some Aspects of Coalescence and Superfractal Interpolation, Ph.D Thesis, Department of Mathematics and Statistics, Indian Institute of Technology, Kanpur, March (2011)

102. Peres, Y., Rams, M., Simon, K., Solomyak, B.: Equivalence of positive Hausdorff measure and the open set condition for self-conformal sets. Proc. Am. Math. Soc. 129, 2689-2699 (2001)

103. Peres, Y., Schlag, W., Solomyak, B.: Sixty years of Bernoulli convolutions. In: Bandt, C., et al. (ed.) Fractal Geometry and Stochastics II. Progress in Probability, vol. 46, pp. 39-46 (2000)

104. Radin, C.: Space tilings and substitutions. Geom. Dedic. 55, 257-264 (1995)

105. Rao, H.: Dual systems of algebraic iterated function systems. Abstracts for International Conference on Advances in Fractals and Related Topics, Hong Kong, December (2012)

106. Rauzy, G.: Nombres algébrique et substitutions. Bull. Soc. Math. France 110, 147-178 (1982)

107. Rota, G.-C., Strang, W.G.: A note on the joint spectral radius. Nederl. Akad. Wet. Proc., Ser. A 63, 379-381 (1960)

108. Ruan, H.J., Su, W.-Y., Yao, K.: Box dimension and fractional integral of linear fractal interpolation functions. J. Approx. Theory 161, 187-197 (2009)

109. Scealy, R.: $V$-variable fractals and interpolation, Ph.D. Thesis, Australian National University (2008)

110. Siderov, N., Solomyak, B.: On the topology of sums in powers of an algebraic number, arXiv:0909.3324v3 (2011)

111. Solomyak, B.: On the 'Mandelbrot set' for pairs of linear maps: the local geometry. Anal. Theory Appl. 20(2), 149-157 (2004)

112. Solomyak, B.: On the 'Mandelbrot set' for pairs of linear maps: asymptotic self-similarity. Nonlinearity 18, 1927-1943 (2005)

113. Solomyak, B.: Dynamics of self-similar tilings. Ergod. Theory Dyn. Syst. 17, 695-738 (1997)

114. Solomyak, B., Xu, H.: On the 'Mandelbrot set' for a pair of linear maps and complex Bernoulli convolutions. Nonlinearity 16, 1733-1749 (2003)

115. Staiger, L.: How large is the set of disjunction sequences. J. Univ. Comput. Sci. 8, 348-362 (2002)

116. Strichartz, R.S.: Wavelets and self-affine tilings. Constructive Approx. 9, 327-346 (1993)

117. Strichartz, R.S.: Differential Equations on Fractals: A Tutorial. Princeton University Press, Princeton (2006)

118. Strichartz, R.S., Teplyaev, A.: Spectral analysis on infinite Sierpinski fractafolds. J. Anal. Math. 119, 255-297 (2012)

119. Thurston, W.P.: Groups, tilings, and finite state automata. AMS Colloquium Lectures, Boulder (1989)

120. Tosan, E., Guerin, E., Baskurt, A.: Design and reconstruction of fractal surfaces. In: IEEE Computer Society (ed) 6th International Conference on Information Visualisation IV 2002, London, UK, July, pp. 311-316 (2002)

121. Tricot, C.: Curves and Fractal Dimension. Springer, New York (1995)

122. Vince, A.: Replicating tessellations. SIAM J. Discrete Math. 6, 501-521 (1993)

123. Vince, A.: Digit tiling of Euclidean space. In: Baake, M., Moody, R. (eds.) Directions in Mathematical Quasicrystals, pp. 329-370 (2000)

124. Vince, A.: Möbius iterated function systems. Trans. Am. Math. Soc. 365, 419-509 (2013)

125. Williams, R.F.: Composition of contractions. Bol. Soc. Brasil. Mat. 2, 55-59 (1971)

126. Wiandt, T.: Liapunov functions for closed relations. J. Diff. Equ. Appl. 14(7), 705-722 (2008)

127. Zajíček, L.: On $\sigma$-porous sets in abstract spaces. Abstr. Appl. Anal. 5, 509-534 (2005) 